\title{
NON-CROSSING PARTITION LATTICES IN FINITE REAL REFLECTION GROUPS
}

\author{
THOMAS BRADY AND COLUM WATT
}

\begin{abstract}
For a finite real reflection group $W$ with Coxeter element $\gamma$ we give a case-free proof that the closed interval, $[I, \gamma]$, forms a lattice in the partial order on $W$ induced by reflection length. Key to this is the construction of an isomorphic lattice of spherical simplicial complexes. We also prove that the greatest element in this latter lattice embeds in the type $W$ simplicial generalised associahedron, and we use this fact to give a new proof that the geometric realisation of this associahedron is a sphere.
\end{abstract}

\section{INTRODUCTION}

Let $W$ be a finite real reflection group. Associated to $W$ is a finite type Artin group or generalised braid group, $A(W)$. Much of the work on finite type Artin groups takes Garside's paper, [14], as its starting point, using the set of fundamental reflections as a generating set for $W$ and a corresponding standard generating set for $A(W)$. Recently it has been shown that when the set of all reflections is used as a generating set for $W$ and a corresponding generating set is used for $A(W)$, a parallel theory can be constructed. In particular, the new positive monoid embeds in $A(W)$ and new $\mathrm{K}(\pi, 1)$ 's for $A(W)$ have been constructed. The larger generating set gives $A(W)$ a second structure as a Garside group. The larger generating set is proposed in [7], but Daan Krammer has used it independently in unpublished work. In the case of the braid group $B_{n}$, where $W$ is the symmetric group $\Sigma_{n}$, the larger generating set coincides with the band generators from [5]. The second Garside structure for $B_{n}$ is described in [2] and the structure for general finite $W$ in [3]. The general construction of $K(A(W), 1)$ 's is described in [11], using ideas from [4].

A central result needed in the development of this parallel theory is that the closed interval, $[I, \gamma]$, bounded by the identity $I$ and a Coxeter element $\gamma$, forms a lattice in the partial order on $W$ induced by reflection length. The lattice property is used to prove the embedding of the positive monoid and the asphericity of the new $\mathrm{K}(\pi, 1)$ 's. Existing proofs of the lattice property use the classification of finite real reflection groups with different methods applied to the different groups. The symmetric group is handled in [8], and the $C_{n}$ and $D_{n}$ groups in [10]. Bessis treats all cases in [3].

Since the lattice in the symmetric group case coincides with the lattice of noncrossing partitions introduced by Kreweras in [16], it has become customary to call

Received by the editors January 27, 2005 and, in revised form, December 17, 2005.

2000 Mathematics Subject Classification. Primary 20F55; Secondary 05E15. 
this lattice, in the case of general $W$, the type $W$ non-crossing partition lattice. The type $C_{n}$ and type $D_{n}$ lattices are studied in [17] and [1], respectively.

In this paper we give a new proof of the lattice property that is independent of the classification of finite real reflection groups. For this purpose we introduce a new simplicial complex which is a geometric model for the partially ordered set $[I, \gamma]$. If $n$ is the rank of $W$, this simplicial complex will lie in the $(n-1)$-sphere, $S^{n-1}$, in $\mathbf{R}^{n}$, and its vertex set will consist of a set of positive roots for $W$.

The layout of the paper is as follows. In Section 2 we motivate the construction we use. In Section 3, we recall definitions and results about the order on the set of roots. We define the simplicial complex $X(\gamma)$ and give an example. We also collect results about the partial order on the orthogonal group. In Section 4 we extend some of the material from [18] and analyse the dot products of roots of $W$ with elements in the $\gamma$ orbits of vectors in the dual basis. In Section 5 we find simple systems for certain subgroups and describe $X(\sigma)$ when $\sigma$ has length two. After some preparatory work in Section 6, we characterise the geometric realisation of $X(\sigma)$ and we prove the lattice property in Section 7 . We conclude in Section 8 by explaining the connection between our construction and the generalised associahedra of [13]. In the process, we give a new proof that the latter is a spherical simplicial complex.

\section{Motivation}

The idea behind the proof of the lattice property can be described without many of the technicalities that appear later. Let $W$ be a finite, irreducible, real reflection group with reflection set $\mathcal{R}$. We recall from [10] the partial order on $W$ given by the reflection length function $l$. For $u, w \in W$ we say

$$
u \leq w \quad \Leftrightarrow \quad l(w)=l(u)+l\left(u^{-1} w\right),
$$

where $l(v)$ is the smallest positive integer $k$ such that $v$ can be written as a product of $k$ reflections from $\mathcal{R}$. Thus $u \leq w$ if and only if there is a shortest factorisation of $u$ as a product of reflections which is a prefix of a shortest factorisation of $w$. Note that $l$ is not the usual length function associated to a simple system for $W$.

We note that the partial order on $W$ is the restriction to $W$ of a partial order on the orthogonal group $O(n)$ which is introduced in [9] and investigated further in [10]. Some of the notation and results from those papers will be used here. In particular, if $A \in O(n)$, we associate to $A$ two subspaces of $\mathbf{R}^{n}$, namely

$$
M(A)=\operatorname{im}(A-I) \quad \text { and } \quad F(A)=\operatorname{ker}(A-I),
$$

which we call the moved space of $A$ and the fixed space of $A$, respectively. We recall that $M(A)=F(A)^{\perp}$ and note that $F(A)=F\left(A^{-1}\right)$. The main result of [9] implies that if $V$ is a subspace of $M(A)$, then there is a unique $B \in O(n)$ satisfying $M(B)=V$ and $B \leq A$. It follows that the map $M$, which associates to an orthogonal transformation $\alpha$ its moved space $M(\alpha)$, restricts to give a poset isomorphism of the interval $[I, \gamma]$ in $W$ onto its image (in the set of subspaces of $\mathbf{R}^{n}$ ). Here the set of subspaces of $\mathbf{R}^{n}$ is partially ordered by inclusion and is, in fact, a lattice whose meet operation is given by subspace intersection. Unfortunately, the set of subspaces of the form $M(\sigma)$ for $\sigma \leq \gamma$ is not closed under intersection. There are many examples of group elements $\alpha, \beta \leq \gamma$ for which there is no element $\delta \leq \gamma$ in $W$ satisfying $M(\delta)=M(\alpha) \cap M(\beta)$. (For example, if $W=A_{3}=\Sigma_{4}$, then the reflections are transpositions and every 4 -cycle is a Coxeter element. Consider 
$\gamma=(1,2,3,4)$ and the elements $\alpha$ and $\beta$ given by

$$
\alpha=(1,3) \gamma=(1,2)(3,4) \text { and } \beta=(2,4) \gamma=(1,4)(2,3) .
$$

Each of $\alpha$ and $\beta$ has a two-dimensional moved space, these moved spaces intersect inside the three-dimensional space $M(\gamma)$ in a one-dimensional subspace, but no transposition precedes both $\alpha$ and $\beta$.)

It was noted in [10] that a group element $\alpha \leq \gamma$ is characterised by its reflection set $S_{\alpha}$, where

$$
S_{\alpha}=\{R \in \mathcal{R} \mid R \leq \alpha\} .
$$

It was also noted there that whenever $\alpha, \beta$ and $\delta$ are elements of the interval $[I, \gamma]$ in $W$ with the properties that $\delta \leq \alpha, \beta$ and $S_{\delta}=S_{\alpha} \cap S_{\beta}$, then $\delta$ must be the greatest lower bound of $\alpha$ and $\beta$ in $W$; that is, if $\tau \in W$ satisfies $\tau \leq \alpha$, $\beta$, then $\tau \leq \delta$. This suggests that reflection sets might have the correct intersection properties. We will see later (Theorem 7.8) that this is in fact the case. To prove this we fix a set of simple roots for $W$ and replace $M(\alpha)$ by the positive cone on the positive roots associated to those reflections which precede $\alpha$. Two surprising things happen: (i) the intersection of the positive cones associated to two elements $\alpha$ and $\beta$ of $[I, \gamma]$ is equal to the positive cone associated to some other element $\delta$ of $[I, \gamma]$, and (ii) this collection of positive cones intersects the unit sphere in a spherical simplicial complex which we denote $X(\gamma)$.

\section{NotATION AND MAIN DEFINITIONS}

Several of the (now standard) case-free proofs of results about irreducible, finite real reflection groups are due to Steinberg [18]. We will use his numbering of the roots of $W$ in the construction of our simplicial complex. Fix a fundamental chamber $C$ with inward unit normals $\alpha_{1}, \ldots, \alpha_{n}$ and let $R_{1}, \ldots, R_{n}$ be the corresponding reflections. Assume that $S_{1}=\left\{\alpha_{1}, \ldots, \alpha_{s}\right\}$ and $S_{2}=\left\{\alpha_{s+1}, \ldots, \alpha_{n}\right\}$ are orthonormal sets (from Lemma 2.2 of [18]). Let $\gamma$ denote the Coxeter element given by $\gamma=R_{1} R_{2} \ldots R_{n}$ and let $h$ denote the order of $\gamma$ in $W$. We note that $M(\gamma)$ is all of $\mathbf{R}^{n}$. As in [18], we set $\rho_{i}=R_{1} R_{2} \ldots R_{i-1} \alpha_{i}$, where the $\alpha$ 's and the $R$ 's are indexed cyclically modulo $n$. We will use the following explicit formulae which are easily verified:

$$
\rho_{i}=\left\{\begin{array}{cl}
\alpha_{i} & \text { for } i=1, \ldots, s, \\
-\gamma\left(\alpha_{i}\right) & \text { for } i=s+1, \ldots, n, \\
\gamma\left(\rho_{i-n}\right) & \text { for } i>n .
\end{array}\right.
$$

It is proved in [18] that the positive roots relative to $C$ are $\rho_{1}, \ldots, \rho_{n h / 2}$ while the negative roots are $\rho_{(n h / 2)+1}, \ldots, \rho_{n h}$. Furthermore the last $n-s$ positive roots are a permutation (possibly trivial) of $S_{2}$. We use $\preceq$ to denote the total order on the set of positive roots $\left\{\rho_{1}, \ldots, \rho_{n h / 2}\right\}$ determined by the subscripts. For each $i$, let $R\left(\rho_{i}\right)$ denote the reflection in $W$ with fixed subspace $\rho_{i}^{\perp}$ which is given by

$$
R\left(\rho_{i}\right)(x)=x-2\left(\rho_{i} \cdot x\right) \rho_{i} .
$$

Note 3.1. If $\rho_{i}, \rho_{i+1}, \ldots, \rho_{i+n-1}$ are $n$ consecutive roots in this ordering, then $R\left(\rho_{i}\right) R\left(\rho_{i+1}\right) \ldots R\left(\rho_{i+n-1}\right)=\gamma^{-1}$.

In what follows, we often consider group elements which precede $\gamma$. For such an element $\sigma$ (i.e. $\sigma \leq \gamma$ ) we let $P_{\sigma}=\left\{\tau_{1}, \tau_{2}, \ldots, \tau_{t}\right\}$ be the set of positive roots whose reflections precede $\sigma$, and we assume that the subscripts are chosen so that $\tau_{i} \preceq \tau_{j}$ whenever $i \leq j$. 
We will use (often without mention) the following facts about spherical simplices. Any linearly independent set of vectors in $\mathbf{R}^{n}$ determines a spherical simplex provided the angle between each pair of vectors is less than $\pi$. For such a set the spherical simplex is obtained by intersecting the unit sphere with the positive cone on those vectors. The spherical simplex determined by the linearly independent set $\left\{v_{1}, v_{2}, \ldots, v_{k}\right\}$ will be denoted by $\left\langle v_{1}, v_{2}, \ldots, v_{k}\right\rangle$. Since any fixed set of positive roots for $W$ lies in a single open halfspace, each linearly independent subset of such a set of positive roots determines a spherical simplex.

Definition 3.2. We define a set, $X=X(\gamma)$, of spherical simplices by declaring that

- the vertex set is $\left\{\rho_{1}, \rho_{2}, \ldots, \rho_{n h / 2}\right\}$,

- an edge joins $\rho_{i}$ to $\rho_{j}$ whenever $i<j$ and $R\left(\rho_{i}\right) R\left(\rho_{j}\right) \leq \gamma^{-1}$ and

- $\left\langle\rho_{i_{1}}, \rho_{i_{2}}, \ldots, \rho_{i_{k}}\right\rangle$ forms a $(k-1)$-simplex if and only if the vertices are distinct and pairwise joined by edges.

For $\sigma \leq \gamma$, let $X(\sigma)$ be the collection of simplices of $X(\gamma)$ whose vertices lie in $P_{\sigma}$.

Note 3.3. It will follow from Lemma 4.8 that a set of $k \leq n$ distinct vertices, $\left\{\rho_{i_{1}}, \rho_{i_{2}}, \ldots, \rho_{i_{k}}\right\}$, with $1 \leq i_{1}<i_{2}<\cdots<i_{k} \leq n h / 2$, determines a $k-1$ simplex of $X$ if and only if

$$
l\left[R\left(\rho_{i_{1}}\right) R\left(\rho_{i_{2}}\right) \ldots R\left(\rho_{i_{k}}\right) \gamma\right]=n-k .
$$

The largest possible dimension for a simplex of $X$ is $n-1$, and at least $n h / 2-n+1$ of these occur by part (c) of Proposition 4.6 and by Lemma 4.8.

A large part of this paper is devoted to proving that $X(\sigma)$ is a spherical simplicial complex for each $\sigma \leq \gamma$ and to characterising its geometric realisation. The case of $H_{3}$ is illustrated in Figure 1 and Figure 2. Figure 1 shows the moved spaces of the elements in $[I, \gamma]$ intersected with the 2 -sphere and projected stereographically onto $\mathbf{R}^{2}$, while the second figure shows the simplicial complex $X(\gamma)$. Note that the arrangement of the two-dimensional moved spaces in the first figure is the image of the Coxeter arrangement under the (non-orthogonal) linear transformation $(\gamma-I)^{-1}$. This follows because, whenever $\gamma=R\left(\sigma_{1}\right) R\left(\sigma_{2}\right) R\left(\sigma_{3}\right)$, then

$$
\begin{aligned}
M\left(R\left(\sigma_{2}\right) R\left(\sigma_{3}\right)\right) & =M\left(R\left(\sigma_{1}\right) \gamma\right) \\
& =\left[F\left(R\left(\sigma_{1}\right) \gamma\right)\right]^{\perp} \\
& =\left[(\gamma-I)^{-1} M\left(R\left(\sigma_{1}\right)\right)\right]^{\perp} \\
& =\left[(\gamma-I)^{-1}\left(\sigma_{1}\right)\right]^{\perp} .
\end{aligned}
$$

The third equality follows from Lemma 3 of [9], since $\gamma$ has a trivial fixed space. Note 3.1 implies that each pair of consecutive positive roots lies in the moved space of some length two element of $[I, \gamma]$. The second figure shows the restriction of the moved spaces to the span of the positive roots within them, producing the spherical simplicial complex $X(\gamma)$ with vertex set coinciding with the set of positive roots.

Let $\left\{\beta_{1}, \ldots, \beta_{n}\right\}$ be the dual basis to $\left\{\alpha_{1}, \ldots, \alpha_{n}\right\}$. Thus

$$
\beta_{i} \cdot \alpha_{j}=\delta_{i j}, \text { for } 1 \leq i, j \leq n, \text { where } \delta_{i j} \text { is the Kronecker delta. }
$$

With a slight change of notation from [18] we define the vectors $\mu_{i}$ for $1 \leq i \leq n h$ by $\mu_{i}=R_{1} R_{2} \ldots R_{i-1} \beta_{i}$, where the $\beta$ 's and the $R$ 's are again indexed cyclically 


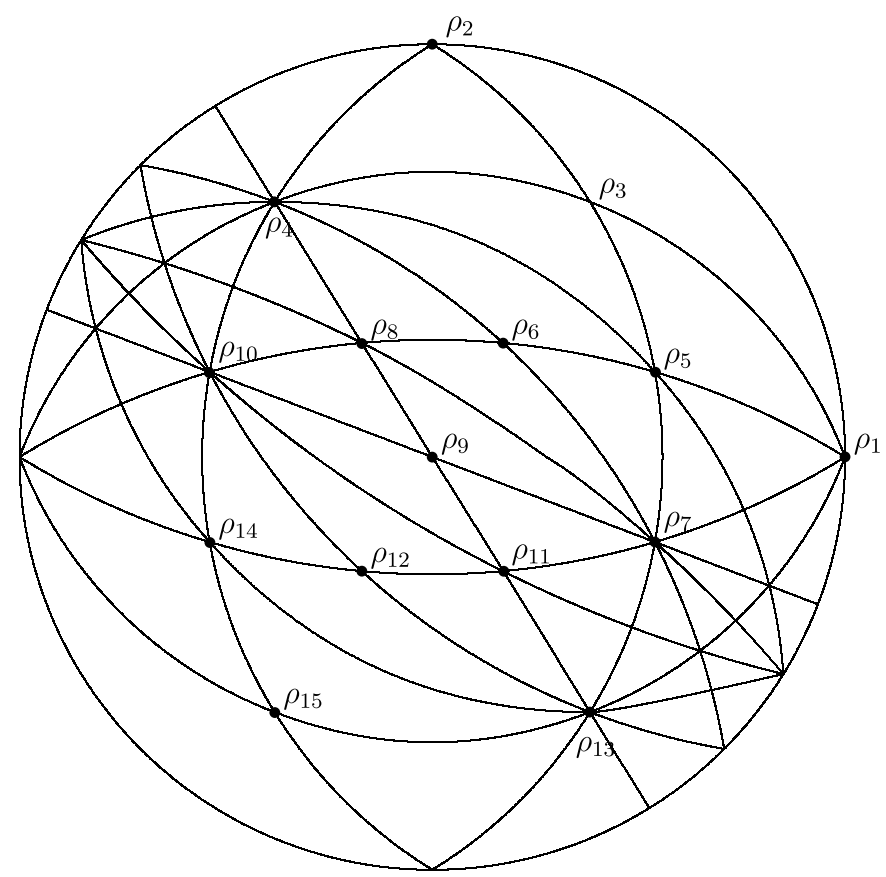

Figure 1

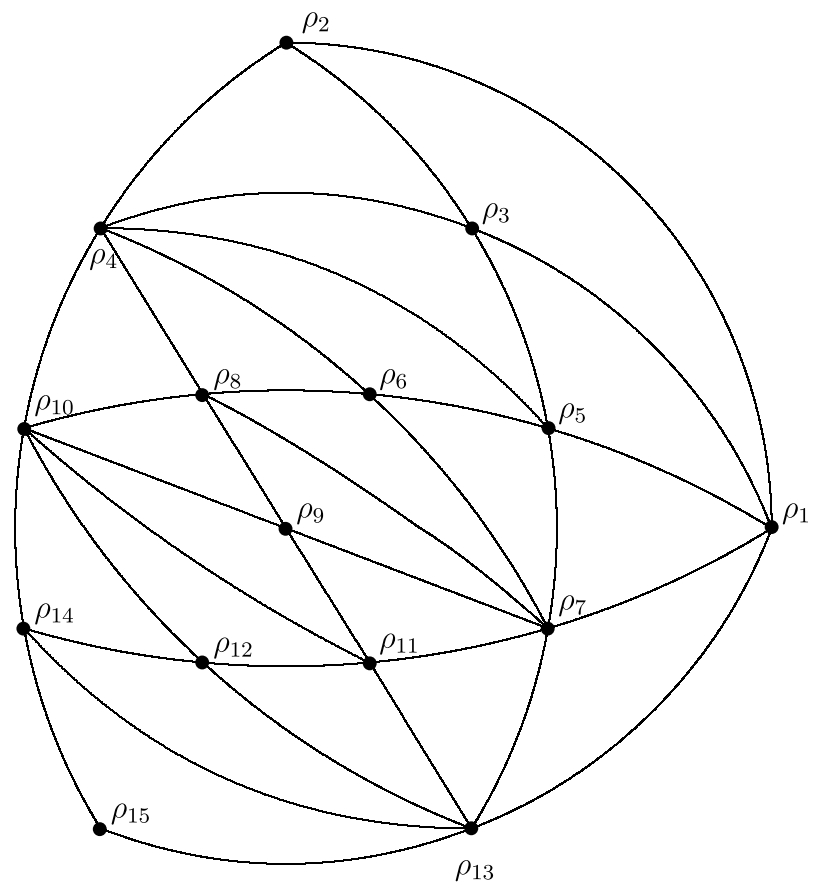

Figure 2 
modulo $n$. As with the $\rho$ 's the following explicit formulae are readily verified:

$$
\mu_{i}= \begin{cases}\beta_{i} & \text { for } i=1, \ldots, n \\ \gamma\left(\mu_{i-n}\right) & \text { for } i>n\end{cases}
$$

If $\sigma$ is an arbitrary root, then we define

$$
\mu(\sigma)=-2(\gamma-I)^{-1} \sigma .
$$

We will see in part (a) of Corollary 4.2 that if $\sigma=\rho_{i}$ for some $i$, then $\mu\left(\rho_{i}\right)=\mu_{i}$.

For convenience, we record some results which will be used in later calculations.

$$
\text { If } \alpha \leq \beta \text {, then } M(\alpha) \subset M(\beta) \text { and } F(\beta) \subset F(\alpha) \text {. }
$$

This equation follows from Corollary 1 of [9].

If $R(\vec{v})$ denotes the reflection in $\vec{v}^{\perp}$, then

$$
\vec{v} \in M(\alpha) \Rightarrow R(\vec{v}) \alpha=\alpha R\left[\alpha^{-1}(\vec{v})\right] .
$$

This equation follows from Property (A-4) of Chapter 1 of [15].

$$
\text { If } \alpha \leq \beta \leq \delta \text {, then } \alpha^{-1} \beta \leq \alpha^{-1} \delta \text { and } \beta \alpha^{-1} \leq \delta \alpha^{-1} \text {. }
$$

The first part of this equation follows from Proposition 3 of [9] while the second part follows from the fact that conjugation in $W$ preserves reflection length.

$$
\text { If } \alpha, \beta \leq \delta \text { and } M(\alpha) \subset M(\beta) \text {, then } \alpha \leq \beta \text {. }
$$

This equation is a consequence of Theorem 1 of [9].

If $l(\alpha)=k$ and $R_{i}$ are reflections, then

$$
\alpha=R_{1} \ldots R_{k} \Rightarrow M\left(R_{j}\right) \subset M\left(R_{1} \alpha\right) \text { for } j=2, \ldots, k .
$$

The preceding equation follows since $R_{1} \alpha$ has length $k-1$ and $R_{1} \alpha=R_{2} \ldots R_{k}$ is a shortest expression for $R_{1} \alpha$ as a product of reflections.

$$
\text { If } \alpha \leq \beta \leq \delta \text {, then } \beta^{-1} \delta \leq \alpha^{-1} \delta \text { and } \delta \beta^{-1} \leq \delta \alpha^{-1} \text {. }
$$

The first inequality follows from

$$
\begin{aligned}
l\left(\alpha^{-1} \delta\right) & =l(\delta)-l(\alpha) \\
& =l(\delta)-l(\beta)+l(\beta)-l(\alpha) \\
& =l\left(\beta^{-1} \delta\right)+l\left(\alpha^{-1} \beta\right) \\
& =l\left(\beta^{-1} \delta\right)+l\left(\delta^{-1} \alpha\left[\alpha^{-1} \beta\right] \alpha^{-1} \delta\right) \\
& =l\left(\beta^{-1} \delta\right)+l\left(\delta^{-1} \beta \alpha^{-1} \delta\right) \\
& =l\left(\beta^{-1} \delta\right)+l\left(\left[\beta^{-1} \delta\right]^{-1} \alpha^{-1} \delta\right),
\end{aligned}
$$

and the second is similar.

If $R_{1}$ and $R_{2}$ are distinct reflections with $R_{1} \leq \alpha$ and $R_{2} \leq \alpha$, then

$$
R_{1} R_{2} \leq \alpha \Leftrightarrow R_{2} \leq R_{1} \alpha \Leftrightarrow R_{1} \leq \alpha R_{2} .
$$

To show (3.7) we note that $R_{1} R_{2} \leq \alpha$ is equivalent to $\alpha=R_{1} R_{2} R_{3} \ldots R_{k}$ for some reflections $R_{3}, \ldots, R_{k}$, where $k=l(\alpha)$. This, in turn, is equivalent to $\alpha=$ $R_{1}\left(R_{3} \ldots R_{k}\right)^{R_{2}} R_{2}$. 


\section{Some GeOMetry of the Root System}

Our later work relies heavily on a detailed analysis of the geometry of the vectors $\rho_{i}$ and $\mu_{i}$. We present the necessary results in this section.

Proposition 4.1. $\gamma\left(\mu_{i}\right)=\mu_{i}-2 \rho_{i}$ for $1 \leq i \leq n h$.

Proof. Because of the recursions satisfied by both $\rho_{i}$ and $\mu_{i}$ it is sufficient to establish this result for $1 \leq i \leq n$. In this case $\mu_{i}=\beta_{i}$ and, since $R_{j}\left(\beta_{i}\right)=\beta_{i}$ if $j \neq i$, we have

$$
\begin{aligned}
\gamma\left(\beta_{i}\right) & =\left(R_{1} \ldots R_{n}\right) \beta_{i} \\
& =\left(R_{1} \ldots R_{i}\right) \beta_{i} \\
& =\left(R_{1} \ldots R_{i-1}\right)\left(\beta_{i}-2 \alpha_{i}\right) \\
& =\beta_{i}-2\left(R_{1} \ldots R_{i-1}\right) \alpha_{i} \\
& =\beta_{i}-2 \rho_{i} \quad \text { as required. }
\end{aligned}
$$

Corollary 4.2. With $\rho_{i}$ and $\mu_{i}$ defined as above we have

(a) $(\gamma-I) \mu_{i}=-2 \rho_{i}$,

(b) $\mu_{i} \cdot \rho_{i}=1$,

(c) $\mu_{i} \in F\left(R\left(\rho_{i}\right) \gamma\right)$.

Proof. Part (a) is immediate from Proposition 4.1. Part (b) follows from Proposition 4.1 and the fact that $\mu_{i} \cdot \mu_{i}=\gamma\left(\mu_{i}\right) \cdot \gamma\left(\mu_{i}\right)$. From Proposition 4.1 and part (b) we see that $\gamma\left(\mu_{i}\right)=R\left(\rho_{i}\right)\left(\mu_{i}\right)$, which is equivalent to statement (c).

Note 4.3. We observe that parts (b) and (c) of Corollary 4.2 characterise $\mu_{i}$ as the unique vector in the one-dimensional subspace $F\left(R\left(\rho_{i}\right) \gamma\right)$ satisfying $\mu_{i} \cdot \rho_{i}=1$.

In what follows we make extensive use of the properties of the matrix of dot products $\left[\mu_{i} \cdot \rho_{j}\right]$. Before establishing the general result we present some examples.

Example 4.4. Consider the symmetry group $A_{2}$ of the equilateral triangle. A simple system for $A_{2}$ is

$$
\alpha_{1}=a(1,-1,0), \alpha_{2}=a(0,1,-1),
$$

where $a=\sqrt{2} / 2$. The dual basis to $\left\{\alpha_{1}, \alpha_{2}\right\}$ is

$$
\beta_{1}=b(2,-1,-1), \beta_{2}=b(1,1,-2),
$$

where $b=\sqrt{2} / 3$ and the dual basis is computed in the 2-dimensional subspace of $\mathbf{R}^{3}$ given by $x+y+z=0$. Using the formula for a reflection we can generate the following table:

\begin{tabular}{|l|l|l|}
\hline$i$ & $\rho_{i}$ & $\mu_{i}$ \\
\hline 1 & $a(1,-1,0)$ & $b(2,-1,-1)$ \\
2 & $a(1,0,-1)$ & $b(1,1,-2)$ \\
3 & $a(0,1,-1)$ & $b(-1,2,-1)$ \\
\hline
\end{tabular}

The entry in the $i^{\text {th }}$ row and $j^{\text {th }}$ column of the following table is $\mu_{i} \cdot \rho_{j}$ :

\begin{tabular}{r|r|r|r|} 
& $\rho_{1}$ & $\rho_{2}$ & $\rho_{3}$ \\
\hline$\mu_{1}$ & 1 & 1 & 0 \\
\hline$\mu_{2}$ & 0 & 1 & 1 \\
\hline$\mu_{3}$ & -1 & 0 & 1 \\
\hline
\end{tabular}


Example 4.5. Consider the symmetry group $C_{3}$ of the cube. A simple system for $C_{3}$ is

$$
\alpha_{1}=(1,0,0), \alpha_{2}=(\sqrt{2} / 2)(0,1,-1), \alpha_{3}=(\sqrt{2} / 2)(-1,0,1) .
$$

The dual basis to $\left\{\alpha_{1}, \alpha_{2}, \alpha_{3}\right\}$ is

$$
\beta_{1}=(1,1,1), \beta_{2}=(0, \sqrt{2}, 0), \beta_{3}=(0, \sqrt{2}, \sqrt{2}) .
$$

As in the preceding example we can generate the following table:

\begin{tabular}{|l|l|l|}
\hline$i$ & $\rho_{i}$ & $\mu_{i}$ \\
\hline 1 & $(1,0,0)$ & $(1,1,1)$ \\
2 & $(\sqrt{2} / 2)(0,1,-1)$ & $\sqrt{2}(0,1,0)$ \\
3 & $(\sqrt{2} / 2)(1,1,0)$ & $\sqrt{2}(0,1,1)$ \\
4 & $(0,1,0)$ & $(-1,1,1)$ \\
5 & $(\sqrt{2} / 2)(1,0,1)$ & $\sqrt{2}(0,0,1)$ \\
6 & $(\sqrt{2} / 2)(0,1,1)$ & $\sqrt{2}(-1,0,1)$ \\
7 & $(0,0,1)$ & $(-1,-1,1)$ \\
8 & $(\sqrt{2} / 2)(-1,1,0)$ & $\sqrt{2}(-1,0,0)$ \\
9 & $(\sqrt{2} / 2)(-1,0,1)$ & $\sqrt{2}(-1,-1,0)$ \\
\hline
\end{tabular}

The entry in the $i^{\text {th }}$ row and $j^{\text {th }}$ column of the following table is $\mu_{i} \cdot \rho_{j}$ :

\begin{tabular}{|r|r|r|r|r|r|r|r|r|}
\hline 1 & 0 & $\sqrt{2}$ & 1 & $\sqrt{2}$ & $\sqrt{2}$ & 1 & 0 & 0 \\
0 & 1 & 1 & $\sqrt{2}$ & 0 & 1 & 0 & 1 & 0 \\
0 & 0 & 1 & $\sqrt{2}$ & 1 & 2 & $\sqrt{2}$ & 1 & 1 \\
-1 & 0 & 0 & 1 & 0 & $\sqrt{2}$ & 1 & $\sqrt{2}$ & $\sqrt{2}$ \\
0 & -1 & 0 & 0 & 1 & 1 & $\sqrt{2}$ & 0 & 1 \\
$-\sqrt{2}$ & -1 & -1 & 0 & 0 & 1 & $\sqrt{2}$ & 1 & 2 \\
-1 & $-\sqrt{2}$ & $-\sqrt{2}$ & -1 & 0 & 0 & 1 & 0 & $\sqrt{2}$ \\
$-\sqrt{2}$ & 0 & -1 & 0 & -1 & 0 & 0 & 1 & 1 \\
$-\sqrt{2}$ & -1 & $-\sqrt{2}$ & $-\sqrt{2}$ & -1 & -1 & 0 & 0 & 1 \\
\hline
\end{tabular}

The second table in each of these examples exhibits certain symmetry properties which are valid in the general case and which we now address.

Proposition 4.6. The quantities $\mu_{i} \cdot \rho_{j}$ have the following properties:

(a) $\mu_{i} \cdot \rho_{j}=-\mu_{j+n} \cdot \rho_{i}$ for all $i$ and $j$.

(b) $\mu_{i} \cdot \rho_{j} \geq 0$, for $1 \leq i \leq j \leq n h / 2$.

(c) $\mu_{i+t} \cdot \rho_{i}=0$, for $1 \leq t \leq n-1$ and for all $i$.

(d) $\mu_{j} \cdot \rho_{i} \leq 0$ for $1 \leq i<j \leq n h / 2$.

Proof. (a) Since $\gamma\left(\mu_{i}\right)=\mu_{i}-2 \rho_{i}$ by Proposition 4.1, we compute

$$
\begin{aligned}
-\mu_{j+n} \cdot \rho_{i} & =-\gamma\left(\mu_{j}\right) \cdot(-1 / 2)\left(\gamma\left(\mu_{i}\right)-\mu_{i}\right) \\
& =1 / 2\left(\gamma\left(\mu_{j}\right) \cdot \gamma\left(\mu_{i}\right)-\gamma\left(\mu_{j}\right) \cdot \mu_{i}\right) \\
& =1 / 2\left(\mu_{j} \cdot \mu_{i}-\gamma\left(\mu_{j}\right) \cdot \mu_{i}\right) \\
& =1 / 2\left(\mu_{j}-\gamma\left(\mu_{j}\right)\right) \cdot \mu_{i} \\
& =\rho_{j} \cdot \mu_{i} .
\end{aligned}
$$


(b) Since $\rho_{p} \cdot \mu_{q}=\gamma\left(\rho_{p}\right) \cdot \gamma\left(\mu_{q}\right)$ we can assume that $1 \leq i \leq n$ and $i<j \leq n h / 2$. The result follows from the fact that $\rho_{j}$ is a positive root and $\mu_{i}$ is part of the dual basis to $\left\{\alpha_{1}, \ldots, \alpha_{n}\right\}$.

(c) Since $\gamma=R\left(\rho_{i+t}\right) R\left(\rho_{i+t-1}\right) \ldots R\left(\rho_{i}\right) \ldots R\left(\rho_{i+t-n+1}\right)$ (by Note 3.1) equation (3.5) implies that $\rho_{i} \cdot \mu_{i+t}=0$.

(d) If $j<i+n$, this follows from part (c). If $j \geq i+n$, this property follows from parts (a) and (b).

Corollary 4.7. If $1 \leq i_{1}<i_{2}<\cdots<i_{m}<k \leq n h / 2$, then $\rho_{k}$ does not lie in the positive cone on $\left\{\rho_{i_{1}}, \ldots, \rho_{i_{m}}\right\}$.

Proof. If the vector $\vec{x}$ is expressible as a non-negative linear combination of the roots $\rho_{i_{1}}, \ldots, \rho_{i_{m}}$, then $\mu_{k} \cdot \vec{x} \leq 0$, since $\mu_{k} \cdot \rho_{i_{j}} \leq 0$ for $j=1, \ldots, m$. As $\mu_{k} \cdot \rho_{k}=1$, the vector $\rho_{k}$ is not expressible in this manner.

The following result provides information about the simplices in $X(\gamma)$.

Lemma 4.8. Suppose $\sigma_{1} \prec \sigma_{2} \prec \cdots \prec \sigma_{k}$ are chosen from the set $\left\{\rho_{1}, \ldots, \rho_{n h / 2}\right\}$. Then the following are equivalent:

(a) $l\left(R\left(\sigma_{1}\right) R\left(\sigma_{2}\right) \ldots R\left(\sigma_{k}\right) \gamma\right)=n-k$.

(b) $\mu\left(\sigma_{i}\right) \cdot \sigma_{j}=0$ whenever $i>j$.

Proof. (a) $\Rightarrow(\mathrm{b})$ : $\quad$ Let $\delta=R\left(\sigma_{1}\right) R\left(\sigma_{2}\right) \ldots R\left(\sigma_{k}\right) \gamma$ and assume that (a) is true. If $i>j$, then repeated application of equation (3.2) gives

$$
\begin{aligned}
\gamma & =R\left(\sigma_{k}\right) \ldots R\left(\sigma_{i}\right) \ldots R\left(\sigma_{j}\right) \ldots R\left(\sigma_{1}\right) \delta \\
& =R\left(\sigma_{i}\right) \ldots R\left(\sigma_{j}\right) \ldots R\left(\sigma_{1}\right) \delta R\left(\gamma^{-1}\left(\sigma_{k}\right)\right) \ldots R\left(\gamma^{-1}\left(\sigma_{i+1}\right)\right) .
\end{aligned}
$$

Since $l(\delta)=n-k$, it follows from equation (3.5) that $R\left(\sigma_{j}\right) \leq R\left(\sigma_{i}\right) \gamma$. Thus $\sigma_{j} \in M\left(R\left(\sigma_{i}\right) \gamma\right)$ and hence $\mu\left(\sigma_{i}\right) \cdot \sigma_{j}=0$ (since $\left.\mu\left(\sigma_{i}\right) \in F\left(R\left(\sigma_{i}\right) \gamma\right)\right)$.

(b) $\Rightarrow$ (a): Assume that (b) is true. Then the $k \times k$ matrix $\left[\mu\left(\sigma_{i}\right) \cdot \sigma_{j}\right]$ is upper triangular with ones on the diagonal (Corollary 4.2 and part (c) of Proposition 4.6). Thus this matrix is non-singular and it follows that each of $\left\{\sigma_{1}, \ldots, \sigma_{k}\right\}$ and $\left\{\mu\left(\sigma_{1}\right), \ldots, \mu\left(\sigma_{k}\right)\right\}$ is a linearly independent set.

Next we show that $R\left(\sigma_{k}\right) R\left(\sigma_{k-1}\right) \ldots R\left(\sigma_{i}\right) \leq \gamma$ by reverse induction on $i$. The case $i=k$ is immediate since $M(\gamma)=\mathbf{R}^{n}$. Now suppose that $i<k$ and that $R\left(\sigma_{k}\right) R\left(\sigma_{k-1}\right) \ldots R\left(\sigma_{i+1}\right) \leq \gamma$. If $i+1 \leq j \leq k$, then using equation (3.6) and equation (3.1) we get

$$
\begin{aligned}
R\left(\sigma_{j}\right) & \leq R\left(\sigma_{k}\right) R\left(\sigma_{k-1}\right) \ldots R\left(\sigma_{i+1}\right) \leq \gamma \\
\Rightarrow R\left(\sigma_{j}\right) \gamma & \geq R\left(\sigma_{i+1}\right) \ldots R\left(\sigma_{k-1}\right) R\left(\sigma_{k}\right) \gamma \\
\Rightarrow F\left[R\left(\sigma_{j}\right) \gamma\right] & \subseteq F\left[R\left(\sigma_{i+1}\right) \ldots R\left(\sigma_{k-1}\right) R\left(\sigma_{k}\right) \gamma\right] \\
\Rightarrow \mu\left(\sigma_{j}\right) & \in F\left[R\left(\sigma_{i+1}\right) \ldots R\left(\sigma_{k-1}\right) R\left(\sigma_{k}\right) \gamma\right],
\end{aligned}
$$

because $\mu\left(\sigma_{j}\right) \in F\left[R\left(\sigma_{j}\right) \gamma\right]$ by part (c) of Corollary 4.2. Thus $\left\{\mu\left(\sigma_{i+1}\right), \ldots, \mu\left(\sigma_{k}\right)\right\}$ is a basis for $F\left[R\left(\sigma_{i+1}\right) \ldots R\left(\sigma_{k-1}\right) R\left(\sigma_{k}\right) \gamma\right]$ (since this subspace has dimension $k-i$ by the inductive hypothesis). As $\sigma_{i}$ is orthogonal to each vector in this basis it follows that

$$
\sigma_{i} \in M\left[R\left(\sigma_{i+1}\right) \ldots R\left(\sigma_{k-1}\right) R\left(\sigma_{k}\right) \gamma\right]
$$

and hence $R\left(\sigma_{k}\right) \ldots R\left(\sigma_{i+1}\right) R\left(\sigma_{i}\right) \leq \gamma$, which completes the inductive step.

Finally, since $l\left(R\left(\sigma_{k}\right) \ldots R\left(\sigma_{2}\right) R\left(\sigma_{1}\right)\right)=k$ (by linear independence of $\sigma_{1}, \ldots, \sigma_{k}$ ) and $R\left(\sigma_{k}\right) \ldots R\left(\sigma_{2}\right) R\left(\sigma_{1}\right) \leq \gamma$, we can conclude that $R\left(\sigma_{1}\right) R\left(\sigma_{2}\right) \ldots R\left(\sigma_{k}\right) \gamma$ has length $n-k$. 


\section{The SIMPle SySTEM FOR $\sigma \leq \gamma$}

In this and the following sections we will consider a fixed element $\sigma \leq \gamma$. As before, we let $P_{\sigma}=\left\{\tau_{1}, \tau_{2}, \ldots, \tau_{t}\right\}$ be the set of positive roots whose reflections precede $\sigma$ and we assume that $\tau_{1} \prec \tau_{2} \prec \cdots \prec \tau_{t}$. In this section we will characterise the simple system associated to $\sigma$ and we will analyse the case where $l(\sigma)=2$.

Let $k=l(\sigma)$. Then $P_{\sigma}$ is the set of positive roots for a (possibly reducible) rank$k$, reflection group $W_{\sigma}=\{\alpha \in W \mid M(\alpha) \subseteq M(\sigma)\}$. (Note that the elements of $W_{\sigma}$ need not necessarily precede $\sigma$.) Let $\Delta=\left\{\delta_{1}, \delta_{2}, \ldots, \delta_{k}\right\}$ be the simple system contained in $P_{\sigma}$ and choose the subscripts so that $\delta_{1} \prec \delta_{2} \prec \cdots \prec \delta_{k}$.

Proposition 5.1. The ordered elements $\delta_{1}, \delta_{2}, \ldots, \delta_{k}$ of $\Delta$ are determined recursively by the fact that $\delta_{i}$ is the last positive root in $M\left(\sigma R\left(\delta_{k}\right) R\left(\delta_{k-1}\right) \ldots R\left(\delta_{i+1}\right)\right)$. In particular, $\delta_{k}=\tau_{t}$.

Proof. Since $\tau_{t}$ is the last root in $P_{\sigma}$ we can apply Corollary 4.7 to $\tau_{t}$ and the set $P_{\sigma}-\left\{\tau_{t}\right\}$ to deduce that $\tau_{t}$ cannot be a positive linear combination of the elements of $P_{\sigma}-\left\{\tau_{t}\right\}$. Thus $P_{\sigma}-\left\{\tau_{t}\right\}$ cannot contain a simple system and $\delta_{k}=\tau_{t}$.

Next we show that $U_{k}=M\left(\sigma R\left(\delta_{k}\right)\right)$ contains $\left\{\delta_{1}, \ldots, \delta_{k-1}\right\}$. To begin, we show that $U_{k}=M(\sigma) \cap \varphi^{\perp}$, where $\varphi=\gamma\left(\mu\left(\delta_{k}\right)\right)$. Note that $U_{k}=M\left(\sigma R\left(\delta_{k}\right)\right)$ has dimension $k-1$. By parts (a) and (b) of Proposition 4.6, if $1 \leq i \leq t$, then

$$
\varphi \cdot \tau_{i}=\gamma\left(\mu\left(\tau_{t}\right)\right) \cdot \tau_{i}=-\tau_{t} \cdot \mu\left(\tau_{i}\right) \leq 0 .
$$

For $i=t$, we obtain $\varphi \cdot \tau_{t}=-1$. Thus $\tau_{t}$ is not an element of $M(\sigma) \cap \varphi^{\perp}$, and this subspace must also have dimension $k-1$. Since $M\left(\sigma R\left(\delta_{k}\right)\right)$ is contained in $M(\sigma)$ and

$$
M\left(\sigma R\left(\delta_{k}\right)\right) \subseteq M\left(\gamma R\left(\delta_{k}\right)\right)=M\left(R\left[\gamma\left(\delta_{k}\right)\right] \gamma\right)=\mu\left(\gamma\left(\delta_{k}\right)\right)^{\perp}=\gamma\left(\mu\left(\delta_{k}\right)\right)^{\perp}
$$

(by equation (3.3) and equation (3.2)), it follows that $U_{k} \subseteq M(\sigma) \cap \varphi^{\perp}$, and hence these subspaces are equal.

Now suppose $\tau$ is an arbitrary element of $P_{\sigma} \cap U_{k}$ and write

$$
\tau=a_{1} \delta_{1}+\cdots+a_{k} \delta_{k}, \text { with each } a_{i} \geq 0 .
$$

Since $\tau \in \varphi^{\perp}$ and $\delta_{i} \cdot \varphi \leq 0$ for $i=1, \ldots, k$, we must have $a_{i}=0$ or $\delta_{i} \cdot \varphi=0$ for each $i$. Thus $\tau$ is a linear combination of those $\delta$ 's in $M(\sigma) \cap \varphi^{\perp}=U_{k}$. However, the span of $P_{\sigma} \cap U_{k}$ is all of $U_{k}$ since $l\left(\sigma R\left(\tau_{t}\right)\right)=k-1$. Hence the $k-1$ simple roots $\delta_{1}, \ldots, \delta_{k-1}$ must lie in $U_{k}$.

The theorem follows since we can now apply the same arguments as above to the shorter element $\sigma^{\prime}=\sigma R\left(\delta_{k}\right)$.

Note 5.2. One can show that $\delta_{i}$ is the first root in $M\left(R\left(\delta_{i-1}\right) \ldots R\left(\delta_{2}\right) R\left(\delta_{1}\right) \sigma\right)$ in an analogous way and, in particular, that $\delta_{1}=\tau_{1}$.

Definition 5.3. We will refer to the intersection with $S^{n-1}$ of the positive cone on $\left\{\delta_{1}, \delta_{2}, \ldots, \delta_{k}\right\}$ as the fat simplex associated to $\sigma$.

Theorem 5.4. If $\sigma \leq \gamma$ has length 2 , then $X(\sigma)$ consists of the $t$ roots from $P_{\sigma}$ and the $(t-1) 1$-cells given by $\left\langle\tau_{i}, \tau_{i+1}\right\rangle$ for $i=1,2, \ldots, t-1$.

Proof. Suppose $\sigma \leq \gamma$ has length two. Since both $R\left(\tau_{i}\right) \sigma$ and $\sigma R\left(\tau_{i}\right)$ have length one for each $i$, each subspace $M(\sigma) \cap \mu\left(\tau_{i}\right)^{\perp}$ contains precisely one positive root $\tau_{j}$, and each $\tau_{j}$ lies in precisely one subspace of the form $M(\sigma) \cap \mu\left(\tau_{i}\right)^{\perp}$. It follows that the $t \times t$ matrix $A=\left[\mu\left(\tau_{i}\right) \cdot \tau_{j}\right]$ has precisely one zero in each row and column. Note 
that the diagonal entries are all 1, the entries below the diagonal are non-positive, while the entries above the diagonal are non-negative (by Proposition 4.6). By Proposition 5.1 and Note 5.2, $\sigma=R\left(\tau_{1}\right) R\left(\tau_{t}\right)$ and hence $\tau_{t} \cdot \mu\left(\tau_{1}\right)=0$. Let $i_{1}>1$ be the value for which $\sigma=R\left(\tau_{i_{1}}\right) R\left(\tau_{1}\right)$. Thus $\mu\left(\tau_{i_{1}}\right) \cdot \tau_{1}=0$ and $X(\sigma)$ contains the edge $\left\langle\tau_{1}, \tau_{i_{1}}\right\rangle$. As row $i_{1}$ contains only one zero and $i_{1} \leq t$, we must have $\mu\left(\tau_{i_{1}}\right) \cdot \tau_{t}>0$ (by Proposition 4.6). Now, since $\left\{\tau_{1}, \tau_{t}\right\}$ is a simple system, we can write each $\tau_{j}$ as a non-negative linear combination of $\tau_{1}$ and $\tau_{t}$. Thus we obtain $\tau_{j} \cdot \mu\left(\tau_{i_{1}}\right) \geq 0$. It follows that each entry in row $i_{1}$ of $A$ is non-negative. Since every row after the second has at least two entries below the diagonal and no more than one of these can be zero, we get $i_{1} \leq 2$. Since we know that $i_{1} \neq 1$ we deduce that $i_{1}$ must be equal to 2 . If $t=2$ the proof is complete.

The rest of the proof uses induction. Assume that $r<t$ and that

$$
\sigma=R\left(\tau_{1}\right) R\left(\tau_{t}\right)=R\left(\tau_{2}\right) R\left(\tau_{1}\right)=\cdots=R\left(\tau_{r}\right) R\left(\tau_{r-1}\right) .
$$

We know that $\sigma=R\left(\tau_{i_{r}}\right) R\left(\tau_{r}\right)$ for some $i_{r}>r$. Thus

$$
\mu\left(\tau_{i_{r}}\right) \cdot \tau_{r}=0 \text { while } \mu\left(\tau_{i_{r}}\right) \cdot \tau_{t}>0 .
$$

If $j \geq r$, then Corollary 4.7 and the fact that $M(\sigma)$ is two-dimensional imply that $\tau_{j}$ can be expressed as a non-negative linear combination of $\tau_{r}$ and $\tau_{t}$. As in the case $r=1$, we obtain $\tau_{j} \cdot \mu\left(\tau_{i_{r}}\right) \geq 0$ for $r \leq j \leq t$. It follows that each of the last $t-r+1$ entries in row $i_{r}$ of $A$ is non-negative. Since row $j$ has $j-1$ entries below the diagonal and no more than one of these can be zero, we get $i_{r} \leq r+1$. Since we know that $i_{r}>r$ we deduce that $i_{r}$ must be equal to $r+1$.

In the case $l(\sigma)=2$, the action of the simple reflections on $P_{\sigma}$ can now be deduced.

Lemma 5.5. If $l(\sigma)=2$ and $P_{\sigma}=\left\{\tau_{1}, \ldots, \tau_{t}\right\}$ is the ordered set of positive roots for reflections preceding $\sigma$, then

$$
\begin{aligned}
R\left(\tau_{1}\right)\left(\tau_{i}\right) & = \begin{cases}\tau_{t-i+2} & \text { for } 2 \leq i \leq t, \\
-\tau_{1} & \text { for } i=1,\end{cases} \\
R\left(\tau_{t}\right)\left(\tau_{i}\right) & = \begin{cases}\tau_{t-i} & \text { for } 1 \leq i \leq t-1, \\
-\tau_{t} & \text { for } i=t .\end{cases}
\end{aligned}
$$

Proof. We will consider the case of $\tau_{1}$. The case of $\tau_{t}$ is similar. From the proof of Theorem 5.4 it follows that the only expressions for $\sigma$ as a product of two reflections are

$$
\sigma=R\left(\tau_{1}\right) R\left(\tau_{t}\right)=R\left(\tau_{2}\right) R\left(\tau_{1}\right)=R\left(\tau_{3}\right) R\left(\tau_{2}\right)=\cdots=R\left(\tau_{t}\right) R\left(\tau_{t-1}\right) .
$$

Since $R\left(\tau_{1}\right)$ permutes $P_{\sigma}-\left\{\tau_{1}\right\}$ (Lemma A in I.4.3 of [15]) and

$$
\sigma=R\left(\tau_{1}\right) R\left(\tau_{t}\right)=\left(R\left(\tau_{1}\right) R\left(\tau_{t}\right) R\left(\tau_{1}\right)\right) R\left(\tau_{1}\right)=R\left(R\left(\tau_{1}\right) \tau_{t}\right) R\left(\tau_{1}\right)
$$

we deduce that $R\left(\tau_{1}\right) \tau_{t}=\tau_{2}$. In general, suppose that $1<i+1 \leq t$ and that $R\left(\tau_{1}\right) \tau_{i+1}=\tau_{t-(i+1)+2}$. Conjugate $R\left(\tau_{t}\right) R\left(\tau_{1}\right)=\sigma^{-1}=R\left(\tau_{i}\right) R\left(\tau_{i+1}\right)$ by $R\left(\tau_{1}\right)$ to obtain

$$
R\left(\tau_{1}\right) R\left(\tau_{t}\right)=\sigma=R\left(R\left(\tau_{1}\right) \tau_{i}\right) R\left(R\left(\tau_{1}\right) \tau_{i+1}\right)=R\left(R\left(\tau_{1}\right) \tau_{i}\right) R\left(\tau_{t-i+1}\right) .
$$

We deduce that $R\left(\tau_{1}\right) \tau_{i}=\tau_{(t-i+1)+1}=\tau_{t-i+2}$ (by comparing with the list of factorisations for $\sigma$ ). Now use reverse induction on $i$, starting at $i=t$. 
Note 5.6. The proofs of Lemma 5.5 and Theorem 5.4 can be shortened considerably by using the notion of a reflection ordering as introduced in [6] and developed in [12]. It follows from the definition of $\rho_{i}$ together with Exercise 6.2 of Chapter 5 of [6] and Proposition 2.13 of [12] that

$$
R\left(\rho_{1}\right), R\left(\rho_{2}\right), \ldots, R\left(\rho_{n h / 2}\right)
$$

is a reflection ordering on $W$, in the sense that the induced order on the set of reflections in any dihedral subgroup is

$$
a<a b a<a b a b a<\cdots<b a b<b,
$$

where $a, b$ is a set of simple reflections for the dihedral group. From this it follows that when $l(\sigma)=2$,

$$
\tau_{2 k+1}=\left[R\left(\tau_{1}\right) R\left(\tau_{t}\right)\right]^{k} \tau_{1} \text { and } \tau_{2 k+2}=\left[R\left(\tau_{1}\right) R\left(\tau_{t}\right)\right]^{k} R\left(\tau_{1}\right) \tau_{t} .
$$

Lemma 5.5 follows immediately from this. The identities

$$
\sigma=R\left(\tau_{1}\right) R\left(\tau_{t}\right)=R\left(\tau_{2}\right) R\left(\tau_{1}\right)=R\left(\tau_{3}\right) R\left(\tau_{2}\right)=\cdots=R\left(\tau_{t}\right) R\left(\tau_{t-1}\right)
$$

also follow from the expressions of $\tau_{i}$ in terms $\tau_{1}$ and $\tau_{t}$, and Theorem 5.4 can be reduced to the argument that the subspaces $M\left(R\left(\tau_{i}\right) \sigma\right)$ and $M\left(\sigma R\left(\tau_{i}\right)\right)$ are onedimensional. However, we prefer to use the current proofs which, although longer, do not require the rather technical facts about reflection orderings.

Lemma 5.7. Assume that $R\left(\rho_{i}\right) R\left(\rho_{j}\right) \leq \gamma$, where $\rho_{i}$ and $\rho_{j}$ are distinct positive roots. Then

(a) $\rho_{i} \cdot \rho_{j} \leq 0$ if $i<j$.

(b) $\rho_{i} \cdot \rho_{j} \geq 0$ if $i>j$.

Proof. Let $P_{\sigma}=\left\{\tau_{1}, \ldots, \tau_{t}\right\}$ be the ordered set of positive roots whose reflections precede $\sigma=R\left(\rho_{i}\right) R\left(\rho_{j}\right)$. By Theorem 5.4, \{ $\left.\tau_{1}, \tau_{t}\right\}$ is the corresponding simple system and the only expressions for $\sigma$ as a product of two reflections are

$$
\sigma=R\left(\tau_{1}\right) R\left(\tau_{t}\right)=R\left(\tau_{2}\right) R\left(\tau_{1}\right)=R\left(\tau_{3}\right) R\left(\tau_{2}\right)=\cdots=R\left(\tau_{t}\right) R\left(\tau_{t-1}\right) .
$$

Since $\sigma=R\left(\tau_{1}\right) R\left(\tau_{t}\right)$ is the only case in which the roots appear in increasing order, if $i<j$, then we must have $\rho_{i}=\tau_{1}$ and $\rho_{j}=\tau_{t}$. Part (a) now follows from $\rho_{i} \cdot \rho_{j}=\tau_{1} \cdot \tau_{t} \leq 0$ (since $\left\{\tau_{1}, \tau_{t}\right\}$ is a simple system).

If $i>j$, then $\rho_{i}=\tau_{r+1}$ and $\rho_{j}=\tau_{r}$ for some $r \geq 1$. Thus we need to verify that $\tau_{i} \cdot \tau_{i+1} \geq 0$ for $i=1, \ldots, t-1$. Using Lemma 5.5 and the facts that reflection is an isometry and $\left\{\tau_{1}, \tau_{t}\right\}$ is a simple system, we obtain

$$
\tau_{1} \cdot \tau_{2}=R\left(\tau_{1}\right) \tau_{1} \cdot R\left(\tau_{1}\right) \tau_{2}=-\tau_{1} \cdot \tau_{t} \geq 0 .
$$

For the same reasons,

$$
\tau_{2} \cdot \tau_{3}=R\left(\tau_{1}\right) R\left(\tau_{t}\right)\left(-\tau_{t}\right) \cdot R\left(\tau_{1}\right) R\left(\tau_{t}\right) \tau_{1}=-\tau_{t} \cdot \tau_{1} \geq 0 .
$$

Finally, if $i \geq 2$, then

$$
\begin{aligned}
\tau_{i} \cdot \tau_{i+1} & =R\left(\tau_{t}\right) R\left(\tau_{1}\right) \tau_{i} \cdot R\left(\tau_{t}\right) R\left(\tau_{1}\right) \tau_{i+1} \\
& =R\left(\tau_{t}\right) \tau_{t-i+2} \cdot R\left(\tau_{t}\right) \tau_{t-i+1}=\tau_{i-2} \cdot \tau_{i-1}
\end{aligned}
$$

and the result follows by induction on $i$. 
Note 5.8. It is immediate from Lemma 5.7 that the edges of $X$ can now be characterised by the following geometric criterion. There is an edge joining the distinct vertices $\rho_{i}$ and $\rho_{j}$ if and only if the vectors subtend a non-obtuse angle and one of $R\left(\rho_{i}\right) R\left(\rho_{j}\right)$ or $R\left(\rho_{j}\right) R\left(\rho_{i}\right)$ precedes $\gamma$.

\section{WALLS OF FAT SIMPLICES}

In this section we continue to investigate a fixed element $\sigma$ of length $k$ which precedes $\gamma$ in $W$. Using the same notation as in the preceding section, we find a dual basis to $\left\{\delta_{1}, \ldots, \delta_{k}\right\}$ (Corollary 6.8 ). We also determine the first top-dimensional simplex of $X(\sigma)$ in the lexicographic order.

Definition 6.1. For each $i=1, \ldots, k$, we define $\epsilon_{i}$ to be the root given by

$$
\epsilon_{i}=\left[R\left(\delta_{1}\right) \ldots R\left(\delta_{i-1}\right)\right]\left(\delta_{i}\right) .
$$

Proposition 6.2. For each $i=1, \ldots, k$, the vector $\epsilon_{i}$ is a positive root. Moreover

$$
\sigma=R\left(\epsilon_{k}\right) R\left(\epsilon_{k-1}\right) \ldots R\left(\epsilon_{1}\right)
$$

and for each $i=1, \ldots, k$

$$
\sigma=R\left(\epsilon_{i}\right) R\left(\delta_{1}\right) \ldots R\left(\delta_{i-1}\right) R\left(\delta_{i+1}\right) \ldots R\left(\delta_{k}\right) .
$$

Proof. The first statement is a special case of Lemma D in Section I.4.3 of [15]. The remaining statements follow from

$$
\begin{aligned}
R\left(\epsilon_{i}\right) & =R\left(\left[R\left(\delta_{1}\right) \ldots R\left(\delta_{i-1}\right)\right]\left(\delta_{i}\right)\right) \\
& =\left[R\left(\delta_{1}\right) R\left(\delta_{2}\right) \ldots R\left(\delta_{i-1}\right)\right] R\left(\delta_{i}\right)\left[R\left(\delta_{i-1}\right) \ldots R\left(\delta_{2}\right) R\left(\delta_{1}\right)\right]
\end{aligned}
$$

since each reflection has order 2 .

Corollary 6.3. The walls of the spherical simplex on the vertices $\left\{\delta_{1}, \ldots, \delta_{k}\right\}$ in the subspace $M(\sigma)$ are given by intersecting the planes $\mu^{\perp}\left(\epsilon_{i}\right)$ with the unit sphere in $M(\sigma)$.

Proof. The second identity in Proposition 6.2 implies that the subspace $\mu^{\perp}\left(\epsilon_{i}\right)$ contains the set $\left\{\delta_{1}, \ldots, \delta_{i-1}, \delta_{i+1}, \ldots, \delta_{k}\right\}$, for $i=1, \ldots, k$.

Note 6.4. We will see in the next section that this simplex is the geometric realisation of $X(\sigma)$.

Definition 6.5. For each $i=1, \ldots, t$, let $\mu^{\prime}\left(\tau_{i}\right)$ be the orthogonal projection of $\mu\left(\tau_{i}\right)$ into $M(\sigma)$.

Proposition 6.6. For each $i, j \in\{1, \ldots, t\}$, we have $\mu^{\prime}\left(\tau_{i}\right) \cdot \tau_{j}=\mu\left(\tau_{i}\right) \cdot \tau_{j}$ and $\mu^{\prime}\left(\tau_{i}\right) \in F\left(R\left(\tau_{i}\right) \sigma\right)$. In particular,

$$
\sigma\left(\mu^{\prime}\left(\tau_{i}\right)\right)=\left[R\left(\tau_{i}\right)\right]\left(\mu^{\prime}\left(\tau_{i}\right)\right)=\mu^{\prime}\left(\tau_{i}\right)-2 \tau_{i} .
$$

Proof. Write $\mu\left(\tau_{i}\right)=\mu^{\prime}\left(\tau_{i}\right)+\vec{y}$, where $\mu^{\prime}\left(\tau_{i}\right) \in M(\sigma)$ and $\vec{y} \in[M(\sigma)]^{\perp}=F(\sigma)$. Then $\mu\left(\tau_{i}\right) \cdot \tau_{j}=\mu^{\prime}\left(\tau_{i}\right) \cdot \tau_{j}$ for any $1 \leq j \leq t$. Now $\mu\left(\tau_{i}\right) \in F\left(R\left(\tau_{i}\right) \gamma\right) \subset F\left(R\left(\tau_{i}\right) \sigma\right)$, because $R\left(\tau_{i}\right) \sigma \leq R\left(\tau_{i}\right) \gamma$ by equation (3.3). But $\vec{y}$ is also an element of $F\left(R\left(\tau_{i}\right) \sigma\right)$, since $F\left(R\left(\tau_{i}\right) \sigma\right)$ contains $F(\sigma)$ by equation (3.1). It follows that $\mu^{\prime}\left(\tau_{i}\right)$ is an element of $F\left(R\left(\tau_{i}\right) \sigma\right)$. The final claim of the proposition follows from this.

Proposition 6.7. For $1 \leq i \leq k$, we have $\mu\left(\epsilon_{i}\right) \cdot \delta_{i}=1$. 
Proof. Fix $i$ and let $\sigma^{\prime}=R\left(\delta_{1}\right) \ldots R\left(\delta_{i}\right)$ (which precedes $\sigma$ and hence $\gamma$ ). Write $\mu\left(\epsilon_{i}\right)=\mu^{\prime \prime}\left(\epsilon_{i}\right)+\vec{z}$, where $\mu^{\prime \prime}\left(\epsilon_{i}\right) \in M\left(\sigma^{\prime}\right)$ and $\vec{z} \in M\left(\sigma^{\prime}\right)^{\perp}=F\left(\sigma^{\prime}\right)$. Applying Proposition 6.6 to $\sigma^{\prime}$ yields

$$
\begin{aligned}
\mu\left(\epsilon_{i}\right) \cdot \delta_{i} & =\sigma^{\prime}\left[\mu\left(\epsilon_{i}\right)\right] \cdot \sigma^{\prime}\left[\delta_{i}\right] \\
& =\sigma^{\prime}\left[\mu^{\prime \prime}\left(\epsilon_{i}\right)+\vec{z}\right] \cdot \sigma^{\prime}\left[\delta_{i}\right] \\
& =\left[\mu^{\prime \prime}\left(\epsilon_{i}\right)-2 \epsilon_{i}+\vec{z}\right] \cdot R\left(\delta_{1}\right) \ldots R\left(\delta_{i-1}\right) R\left(\delta_{i}\right)\left[\delta_{i}\right] \\
& =\left[\mu\left(\epsilon_{i}\right)-2 \epsilon_{i}\right] \cdot R\left(\delta_{1}\right) \ldots R\left(\delta_{i-1}\right)\left[-\delta_{i}\right] \\
& =\left[\mu\left(\epsilon_{i}\right)-2 \epsilon_{i}\right] \cdot\left[-\epsilon_{i}\right] \\
& =1 \text { as required. }
\end{aligned}
$$

The following corollary is immediate from Corollary 6.3 and Proposition 6.7

Corollary 6.8. The dual basis to $\left\{\delta_{1}, \ldots, \delta_{k}\right\}$ is $\left\{\mu^{\prime}\left(\epsilon_{1}\right), \ldots, \mu^{\prime}\left(\epsilon_{k}\right)\right\}$.

It need not be the case that $\epsilon_{1} \prec \ldots \prec \epsilon_{k}$. However the induced order on this set still determines a factorisation of $\sigma$ (Proposition 6.11 below). First we make a definition.

Definition 6.9. Let $\theta_{1}, \ldots, \theta_{k}$ be the reordering of $\epsilon_{1}, \ldots, \epsilon_{k}$ for which $\theta_{i} \prec \theta_{j}$ whenever $i<j$.

Lemma 6.10. If $i<j$ but $\epsilon_{i}>\epsilon_{j}$, then $R\left(\epsilon_{j}\right) R\left(\epsilon_{i}\right)=R\left(\epsilon_{i}\right) R\left(\epsilon_{j}\right)$.

Proof. Assume that $i<j$ and that $\epsilon_{i}>\epsilon_{j}$. We need to show that $\epsilon_{i} \cdot \epsilon_{j}=0$. Since $\left\{\delta_{1}, \ldots, \delta_{k}\right\}$ is a simple system, each of the dot products $\delta_{i} \cdot \delta_{j}$ is non-positive for $i \neq j$. It follows that $\left[R\left(\delta_{j-1}\right)\right]\left(\delta_{j}\right)$ is a non-negative linear combination of $\delta_{j-1}$ and $\delta_{j}$ and, by induction, that $\left[R\left(\delta_{i+1}\right) \ldots R\left(\delta_{j-1}\right)\right]\left(\delta_{j}\right)$ is a non-negative linear combination of $\delta_{i+1}, \delta_{i+2}, \ldots, \delta_{j}$. Hence, $\delta_{i} \cdot\left[R\left(\delta_{i+1}\right) \ldots R\left(\delta_{j-1}\right)\right]\left(\delta_{j}\right) \leq 0$. Now, since $i<j$, we can compute that

$$
\begin{aligned}
\epsilon_{i} \cdot \epsilon_{j} & =\left[R\left(\delta_{1}\right) \ldots R\left(\delta_{i-1}\right)\right]\left(\delta_{i}\right) \cdot\left[R\left(\delta_{1}\right) \ldots R\left(\delta_{j-1}\right)\right]\left(\delta_{j}\right) \\
& =\delta_{i} \cdot\left[R\left(\delta_{i}\right) \ldots R\left(\delta_{j-1}\right)\right]\left(\delta_{j}\right) \\
& =\left[R\left(\delta_{i}\right)\right]\left(\delta_{i}\right) \cdot\left[R\left(\delta_{i+1}\right) \ldots R\left(\delta_{j-1}\right)\right]\left(\delta_{j}\right) \\
& =-\delta_{i} \cdot\left[R\left(\delta_{i+1}\right) \ldots R\left(\delta_{j-1}\right)\right]\left(\delta_{j}\right) \\
& \geq 0
\end{aligned}
$$

However, since $i<j$, the identity $\sigma=R\left(\epsilon_{k}\right) \ldots R\left(\epsilon_{j}\right) \ldots R\left(\epsilon_{i}\right) \ldots R\left(\epsilon_{1}\right)$ implies that $R\left(\epsilon_{j}\right) R\left(\epsilon_{i}\right)$ precedes $\sigma$ and hence $\gamma$. Now part (a) of Lemma 5.7 implies that $\epsilon_{i} \cdot \epsilon_{j} \leq 0$ (because $\epsilon_{i}>\epsilon_{j}$ ). We conclude that $\epsilon_{i} \cdot \epsilon_{j}=0$, as required.

Proposition 6.11. The elements $\theta_{1}, \ldots, \theta_{k}$ satisfy the identity

$$
\sigma=R\left(\theta_{k}\right) \ldots R\left(\theta_{1}\right) .
$$

Proof. We know that $\sigma=R\left(\epsilon_{k}\right) \ldots R\left(\epsilon_{1}\right)$. If $\epsilon_{i}=\theta_{i}$ for each $i$, there is nothing to prove. Otherwise, repeated application of Lemma 6.10 yields the required result.

Corollary 6.12. The simplex $\left\langle\theta_{1}, \ldots, \theta_{k}\right\rangle$ is the first top dimensional simplex of $X(\sigma)$ in the lexicographic order. 
Proof. Since $\theta_{1}<\theta_{2}<\cdots<\theta_{k}$ and $\sigma=R\left(\theta_{k}\right) \ldots R\left(\theta_{1}\right)$, the $(k-1)$-simplex $\left\langle\theta_{1}, \ldots, \theta_{k}\right\rangle$ is in $X(\sigma)$. As $\left\{\delta_{1}, \ldots, \delta_{k}\right\}$ is the simple system corresponding to $P_{\sigma}$, $\left\{\theta_{1}, \ldots, \theta_{k}\right\}$ is a rearrangement of $\left\{\epsilon_{1}, \ldots, \epsilon_{k}\right\}$ and

$$
\mu\left(\epsilon_{a}\right) \cdot \delta_{b}= \begin{cases}0 & \text { for } a \neq b, \\ 1 & \text { for } a=b,\end{cases}
$$

it follows that $\mu\left(\theta_{i}\right) \cdot \tau_{j} \geq 0$ for $1 \leq i \leq k$ and $1 \leq j \leq t$. However, Proposition 4.6 implies that whenever $\tau_{j}<\theta_{i}$, the dot product $\mu\left(\theta_{i}\right) \cdot \tau_{j}$ is non-positive, and hence it must be zero. Therefore

$$
\left\{\tau_{j} \mid \tau_{j}<\theta_{i}\right\} \subseteq M(\sigma) \cap \mu\left(\theta_{i}\right)^{\perp} \cap \mu\left(\theta_{i+1}\right)^{\perp} \cap \cdots \cap \mu\left(\theta_{k}\right)^{\perp}
$$

which is an $(i-1)$-dimensional subspace of $\mathbf{R}^{n}$ since the $\theta$ 's are linearly independent. Now if $\left\langle\tau_{i_{1}}, \ldots, \tau_{i_{k}}\right\rangle$ is a $(k-1)$-simplex of $X(\sigma)$ with $\tau_{i_{1}}<\tau_{i_{2}}<\cdots<\tau_{i_{k}}$, then $\left\langle\tau_{i_{1}}, \ldots, \tau_{i_{j}}\right\rangle$ is a $(j-1)$-simplex for each $j \leq k$, and this forces $\tau_{i_{j}} \geq \theta_{j}$.

We finish this section with two examples. The first shows that the first $k$ roots of $P_{\sigma}$ may fail to span a top dimensional simplex. The second example illustrates that even in the case $\sigma=\gamma$, it is possible that there is some $i<j$ for which $\epsilon_{j} \prec \epsilon_{i}$.

Example 6.13. Consider the symmetry group of the 4-dimensional cube. One simple system of unit vectors for this group is

$$
\begin{gathered}
\alpha_{1}=(1,0,0,0), \alpha_{2}=(\sqrt{2} / 2)(0,1,0,-1), \\
\alpha_{3}=(\sqrt{2} / 2)(-1,0,0,1), \alpha_{4}=(\sqrt{2} / 2)(0,-1,1,0) .
\end{gathered}
$$

The element $\gamma=[1,2,3,4]$ (in the notation of [10]) is one of the Coxeter elements determined by this simple system, where

$$
[1,2,3,4](x, y, z, w)=(-w, x, y, z) .
$$

The element $\sigma=[1,2,3]$ precedes $\gamma$, has length three and its first three positive roots are

$$
(1,0,0,0), \quad(\sqrt{2} / 2)(1,1,0,0), \quad(0,1,0,0) .
$$

These cannot span a 2-simplex since they are linearly dependent.

Example 6.14. Consider the group $A_{3}=\Sigma_{4}$. We have seen that the four-cycle $\gamma=(1,2,3,4)$ is a Coxeter element. The simple system corresponding to the factorisation $\gamma=(1,3)(1,2)(3,4)$ is given by normalising

$$
(1,0,-1,0), \quad(-1,1,0,0), \quad(0,0,1,-1) .
$$

For notational convenience we will identify roots with the transpositions they determine. The global order is the following:

$$
(1,3),(2,3),(1,4),(2,4),(3,4),(1,2) .
$$

We use Proposition 5.1 to find the simple system. First $\delta_{3}=(1,2)$ since this is the last root. Since $(1,2,3,4)(1,2)=(1,3,4), \delta_{2}=(3,4)$. Finally, $\delta_{1}=(1,3)$. Thus $\epsilon_{1}=(1,3)=\tau_{1}, \epsilon_{2}=(1,4)=\tau_{3}$ and $\epsilon_{3}=(2,3)=\tau_{2}$ so that $\epsilon_{1} \prec \epsilon_{3} \prec \epsilon_{2}$. 


\section{Characterisation of $|X(\sigma)|$ and proof of the lattice property}

In this section we show that if $\sigma \leq \gamma$, then $X(\sigma)$ is a simplicial complex. We characterise the geometric realisation $|X(\sigma)|$ of the subcomplex $X(\sigma)$ and use this characterisation to prove that the interval $[I, \gamma]$ in $W$ is a lattice. Throughout this section we continue to use the notation of the earlier sections.

We begin with a technical result (Proposition 7.2) about the separation properties of the hyperplanes $\left\{\mu\left(\tau_{i}\right)^{\perp}\right\}$ which is used in the proof of Proposition 7.6. It depends on Proposition 7.1 which is stated for convenient reference and which concerns the action of $\sigma^{-1}$ on $P_{\sigma}$. Using the factorisation $\sigma^{-1}=R\left(\delta_{k}\right) \ldots R\left(\delta_{1}\right)$, this is a special case of Theorem B in Section I.4.3 of [15].

Proposition 7.1. If $\tau_{s} \in P_{\sigma}$, then $\sigma^{-1}\left(\tau_{s}\right) \in-P_{\sigma}$ if and only if $\tau_{s}=\epsilon_{i}$ for some $i$ with $1 \leq i \leq k$.

Proposition 7.2. Let $\tau_{a}$ and $\tau_{s}$ be elements of $P_{\sigma}$ such that $\tau_{a} \succeq \theta_{k}, \tau_{s} \prec \tau_{a}$, $\tau_{s} \notin\left\{\theta_{1}, \theta_{2}, \ldots, \theta_{k-1}, \theta_{k}\right\}$ and $\tau_{a} \in \mu\left(\tau_{s}\right)^{\perp}$. Then we can find two roots $\tau_{b}$ and $\tau_{c}$ in $P_{\sigma}$ with $\tau_{b}, \tau_{c} \prec \tau_{a}$, which both lie on the hyperplane $\mu\left(\tau_{a}\right)^{\perp}$, but which are separated by the hyperplane $\mu\left(\tau_{s}\right)^{\perp}$.

Proof. Since $\tau_{a} \cdot \mu\left(\tau_{s}\right)=0, \tau_{a} \in M\left(R\left(\tau_{s}\right) \gamma\right)$ and the element $R\left(\tau_{s}\right) R\left(\tau_{a}\right)$ precedes $\gamma$ by equation (3.7).

Construction of $\tau_{b}$ : Let $Q=\left\{\tau_{i} \in P_{\sigma} \mid R\left(\tau_{i}\right) \leq R\left(\tau_{s}\right) R\left(\tau_{a}\right)\right\}$. Since $\tau_{s} \prec \tau_{a}$, the proof of Lemma 5.7 implies that (i) the set $\left\{\tau_{s}, \tau_{a}\right\}$ is a simple system which spans $Q$ and (ii) $\tau_{s}$ is the first root and $\tau_{a}$ the last root of $Q$. By Lemma 5.5, the element $\tau_{b}$ given by $\tau_{b}=\left[R\left(\tau_{a}\right)\right]\left(\tau_{s}\right)$ is in $Q$ and $s<b<a$. Since

$$
R\left(\tau_{a}\right) R\left(\tau_{b}\right)=R\left(\tau_{a}\right)\left(R\left(\tau_{a}\right) R\left(\tau_{s}\right) R\left(\tau_{a}\right)\right)=R\left(\tau_{s}\right) R\left(\tau_{a}\right) \leq \gamma,
$$

we deduce that $\tau_{b} \cdot \mu\left(\tau_{a}\right)=0$. Finally $\tau_{b} \cdot \mu\left(\tau_{s}\right)>0$ because

$$
\mu\left(\tau_{s}\right) \cdot \tau_{b}=\mu\left(\tau_{s}\right) \cdot\left[R\left(\tau_{a}\right)\right]\left(\tau_{s}\right)=\mu\left(\tau_{s}\right) \cdot\left\{\tau_{s}-2\left(\tau_{a} \cdot \tau_{s}\right) \tau_{a}\right\}=1
$$

since $\mu\left(\tau_{s}\right) \cdot \tau_{s}=1$ and $\mu\left(\tau_{s}\right) \cdot \tau_{a}=0$.

Construction of $\tau_{c}$ : As $\tau_{s}$ is not an element of $\left\{\theta_{1}, \ldots, \theta_{k}\right\}=\left\{\epsilon_{1}, \ldots, \epsilon_{k}\right\}$, the root $\tau_{c}$ defined by $\tau_{c}=\sigma^{-1}\left(\tau_{s}\right)$ is an element of $P_{\sigma}$ (by Proposition 7.1). Since $R\left(\tau_{s}\right) R\left(\tau_{a}\right) \leq \gamma$ and $M\left[R\left(\tau_{s}\right) R\left(\tau_{a}\right)\right] \subset M(\sigma)$ we deduce by equation (3.4) that $R\left(\tau_{s}\right) R\left(\tau_{a}\right) \leq \sigma$. This gives $R\left(\tau_{a}\right) \leq R\left(\tau_{s}\right) \sigma=\sigma R\left(\tau_{c}\right)$ by equation (3.2) and hence $R\left(\tau_{a}\right) R\left(\tau_{c}\right) \leq \sigma \leq \gamma$ by equation (3.7). It follows that $\tau_{c} \cdot \mu\left(\tau_{a}\right)=0$. Now $\tau_{c} \cdot \mu\left(\tau_{s}\right)<0$ since

$$
\begin{aligned}
\mu\left(\tau_{s}\right) \cdot \tau_{c} & =\mu^{\prime}\left(\tau_{s}\right) \cdot \tau_{c} \\
& =\mu^{\prime}\left(\tau_{s}\right) \cdot \sigma^{-1}\left(\tau_{s}\right) \\
& =\sigma\left(\mu^{\prime}\left(\tau_{s}\right)\right) \cdot \tau_{s} \\
& =\left\{\mu^{\prime}\left(\tau_{s}\right)-2 \tau_{s}\right\} \cdot \tau_{s} \text { by Proposition } 6.6 \\
& =1-2=-1 .
\end{aligned}
$$

As $\mu\left(\tau_{s}\right) \cdot \tau_{c}<0$, Proposition 4.6 implies that $\tau_{c} \prec \tau_{s}$ and hence $\tau_{c} \prec \tau_{a}$.

Definition 7.3. If $\tau_{i}$ is a root in $P_{\sigma}$ and $\rho$ is any positive root, we define

- $\mu\left(\tau_{i}\right)^{+}=\left\{x \in \mathbf{R}^{\mathbf{n}} \mid x \cdot \mu\left(\tau_{i}\right) \geq 0\right\}$ (a positive halfspace),

- $\mu\left(\tau_{i}\right)^{-}=\left\{x \in \mathbf{R}^{\mathbf{n}} \mid x \cdot \mu\left(\tau_{i}\right) \leq 0\right\}$ (a negative halfspace), 
- $X(\sigma, \rho)=$ the set of simplices of $X$ whose vertices both lie in $M(\sigma)$ and precede $\rho$ in the total order,

- $c[X(\sigma, \rho)]=$ the positive cone on the set $|X(\sigma, \rho)|$,

- $Y(\sigma, \rho)=$ the positive cone on those roots which both lie in $M(\sigma)$ and precede $\rho$ in the total order,

- $Z\left(\sigma, \tau_{i}\right)=M(\sigma) \cap \mu\left(\theta_{1}\right)^{+} \cap \cdots \cap \mu\left(\theta_{k}\right)^{+} \cap \mu\left(\tau_{i+1}\right)^{-} \cap \cdots \cap \mu\left(\tau_{t}\right)^{-}$, for $\tau_{i} \geq \theta_{k}$.

Proposition 7.4. For each $i=1, \ldots, t$, the set $X\left(\sigma, \tau_{i}\right)$ is a simplicial complex.

Proof. We use induction on $i$. First note that $X\left(\sigma, \tau_{1}\right)=\left\{\left\langle\tau_{1}\right\rangle\right\}$, a zero-dimensional simplicial complex.

Assume now that $i \geq 1$ and that $X\left(\sigma, \tau_{i}\right)$ is a simplicial complex. By definition, if $\tau_{j} \in X\left(\sigma, \tau_{i}\right)$, then $\left\langle\tau_{j}, \tau_{i+1}\right\rangle \in X\left(\sigma, \tau_{i+1}\right)$ if and only if $R\left(\tau_{i+1}\right) R\left(\tau_{j}\right) \leq \gamma$ since $\tau_{j} \prec \tau_{i+1}$. However, $R\left(\tau_{i+1}\right) R\left(\tau_{j}\right) \leq \gamma$ if and only if $\tau_{j} \cdot \mu\left(\tau_{i+1}\right)=0$, because $\mu\left(\tau_{i+1}\right) \in F\left(R\left(\tau_{j}\right) \gamma\right)$. It follows from Proposition 4.6 that the only vertices of $X\left(\sigma, \tau_{i}\right)$ that are not joined to $\tau_{i+1}$ by an edge in $X\left(\sigma, \tau_{i+1}\right)$, lie in the interior of the halfspace $\mu\left(\tau_{i+1}\right)^{+}$. Hence, $\mu\left(\tau_{i+1}\right)^{\perp} \cap\left|X\left(\sigma, \tau_{i}\right)\right|$ is a simplicial complex. Now each simplex in $X\left(\sigma, \tau_{i+1}\right) \backslash X\left(\sigma, \tau_{i}\right)$ is of the form $\left\langle\tau_{a_{1}}, \ldots, \tau_{a_{b}}, \tau_{i+1}\right\rangle$ where $\tau_{a_{1}} \prec \cdots \prec \tau_{a_{b}} \prec \tau_{i+1}$ and where $\tau_{a_{c}} \in \mu\left(\tau_{i+1}\right)^{\perp}$ for $c=1, \ldots, b$. Thus the simplex $\left\langle\tau_{a_{1}}, \ldots, \tau_{a_{b}}\right\rangle$ of $X\left(\sigma, \tau_{i}\right)$ is contained in $\mu\left(\tau_{i+1}\right)^{\perp}$. Using this, it is straightforward to verify that the intersection of any two simplices in $X\left(\sigma, \tau_{i+1}\right)$ is itself a simplex in $X\left(\sigma, \tau_{i+1}\right)$.

Corollary 7.5. For each $\sigma \leq \gamma, X(\sigma)$ is a simplicial complex of dimension $l(\sigma)-1$. In particular, $X(\gamma)$ is a simplicial complex of dimension $n-1$.

Proposition 7.6. For $\theta_{k} \preceq \tau_{i} \preceq \tau_{t}$, we have $c\left[X\left(\sigma, \tau_{i}\right)\right]=Y\left(\sigma, \tau_{i}\right)=Z\left(\sigma, \tau_{i}\right)$.

Proof. It suffices to show that $Z\left(\sigma, \tau_{i}\right)$ is contained in $c\left[X\left(\sigma, \tau_{i}\right)\right]$ because $c[X(\sigma, \rho)]$ is contained in $Y(\sigma, \rho)$ (by definition) and $Y\left(\sigma, \tau_{i}\right)$ is contained in $Z\left(\sigma, \tau_{i}\right)$ when $\tau_{i} \succeq \theta_{k}$ (by Proposition 4.6). The proof is by induction on $i$, starting at the value $i_{0}$ for which $\tau_{i_{0}}=\theta_{k}$.

Base step: Let $F_{0}=X\left(\sigma, \tau_{i_{0}-1}\right)$ be the subcomplex of $X(\gamma)$ whose vertex set is $\left\{\tau_{1}, \tau_{2}, \ldots, \tau_{i_{0}-1}\right\}$. Since $\left\langle\theta_{1}, \theta_{2}, \ldots, \theta_{k}\right\rangle \in X(\sigma)$ (by Corollary 6.12) and $\theta_{1}, \theta_{2}, \ldots, \theta_{k-1} \in F_{0}$, it follows that the simplex $\left\langle\theta_{1}, \theta_{2}, \ldots, \theta_{k-1}\right\rangle$ is in $F_{0}$. Furthermore, if $\sigma_{0}=\left[R\left(\theta_{k}\right)\right](\sigma)$, then $F_{0}=X\left(\sigma_{0}, \tau_{i_{0}-1}\right)$. By induction on $k=l(\sigma)$, we can assume that the assertion of the theorem is valid if $\sigma$ is replaced by the length $k-1$ element $\sigma_{0}$ and hence $c\left[X\left(\sigma_{0}, \tau_{i_{0}-1}\right)\right]=Z\left(\sigma_{0}, \tau_{i_{0}-1}\right)$. (The base case of this inner induction is trivial since $k=1$ corresponds to a rank 1 group.) Thus $\left|F_{0}\right|$ is convex and $(k-2)$-dimensional.

Now let $V_{0}=X\left(\sigma, \tau_{i_{0}}\right)$, which has vertex set $\left\{\tau_{1}, \tau_{2}, \ldots, \tau_{i_{0}}\right\}$. Since $\mu\left(\theta_{k}\right) \cdot \tau_{j}=0$ whenever $\tau_{j} \prec \theta_{k}$ (as in the proof of Corollary 6.12 ), it follows that $\left|V_{0}\right|$ is the cone with base $\left|F_{0}\right|$ and apex $\tau_{i_{0}}$. Thus $\left|V_{0}\right|$ is convex and $(k-1)$-dimensional.

The containment of $Z\left(\sigma, \tau_{i_{0}}\right)$ in $c\left[X\left(\sigma, \tau_{i_{0}}\right)\right]$ is demonstrated by examining the supports of the facets of the positive cone, $c\left[V_{0}\right]$, on $\left|V_{0}\right|$. Each support is of the form $M(\sigma) \cap \mu\left(\tau_{j}\right)^{\perp}$ for some $\tau_{j}$.

One of the facets of $c\left[V_{0}\right]$ contains $\left|F_{0}\right|$ and hence it has support $M(\sigma) \cap \mu\left(\tau_{i_{0}}\right)^{\perp}$. Each of the other facets of $c\left[V_{0}\right]$ contains the vertex $\tau_{i_{0}}=\theta_{k}$ and hence its support is of the form $M(\sigma) \cap \mu\left(\tau_{j}\right)^{\perp}$ for some $j \neq i_{0}$. In fact, $\tau_{j}$ must belong to either $\left\{\theta_{1}, \ldots, \theta_{k-1}\right\}$ or $\left\{\tau_{j} \mid \tau_{j} \succ \theta_{k}\right\}$ in this case. For $\mu\left(\tau_{j}\right)^{\perp}$ cannot separate $\left|F_{0}\right|$ (because $\left|F_{0}\right| \subset\left|V_{0}\right|$ which is contained in one of the half-spaces bounded by $\mu\left(\tau_{j}\right)^{\perp}$ ) 
and hence if $\tau_{j} \notin\left\{\theta_{1}, \ldots, \theta_{k-1}\right\}$, then Proposition 7.2 implies that we must have $\tau_{j} \succ \theta_{k}$. Thus the set of facets of $c\left[V_{0}\right]$ is of the form

$$
\left\{M(\sigma) \cap \mu\left(\tau_{j}\right)^{\perp} \mid \tau_{j} \in\left\{\theta_{i_{1}}, \ldots, \theta_{i_{a}}, \theta_{k}\right\} \cup\left\{\tau_{j_{1}}, \ldots, \tau_{j_{b}}\right\}\right\}
$$

where $j_{l}>i_{0}$ for $l=1,2, \ldots, b$. It follows that

$$
\begin{aligned}
Z\left(\sigma, \tau_{i_{0}}\right) & =M(\sigma) \cap \mu\left(\theta_{1}\right)^{+} \cap \cdots \cap \mu\left(\theta_{k}\right)^{+} \cap \mu\left(\tau_{i_{0}+1}\right)^{-} \cap \cdots \cap \mu\left(\tau_{t}\right)^{-} \\
& \subseteq M(\sigma) \cap \mu\left(\theta_{i_{1}}\right)^{+} \cap \cdots \cap \mu\left(\theta_{i_{a}}\right)^{+} \cap \mu\left(\theta_{k}\right)^{+} \\
& \cap \mu\left(\tau_{j_{1}}\right)^{-} \cap \cdots \cap \mu\left(\tau_{j_{b}}\right)^{-} \\
= & c\left[X\left(\sigma, \tau_{i_{0}}\right)\right] .
\end{aligned}
$$

Inductive step: Assume now that $i \geq i_{0}$ and that $c\left[X\left(\sigma, \tau_{i}\right)\right]=Z\left(\sigma, \tau_{i}\right)$. Let $F$ and $V$ be the subcomplexes of $X(\gamma)$ whose sets of vertices are

$$
\left\{\tau_{j} \mid 1 \leq j \leq i \text { and } \mu\left(\tau_{i+1}\right) \cdot \tau_{j}=0\right\}
$$

and

$$
\left\{\tau_{i+1}\right\} \cup\left\{\tau_{j} \mid 1 \leq j \leq i \text { and } \mu\left(\tau_{i+1}\right) \cdot \tau_{j}=0\right\},
$$

respectively. Then $|V|$ is a cone with base $|F|$ and apex $\tau_{i+1}$. We prove that the closure, $Z$, of $Z\left(\sigma, \tau_{i+1}\right) \backslash Z\left(\sigma, \tau_{i}\right)$ is contained in the positive cone, $c[V]$, on $|V|$. Since $|V|$ is contained in $\left|X\left(\sigma, \tau_{i+1}\right)\right|$ and $Z\left(\sigma, \tau_{i+1}\right)=Z \cup Z\left(\sigma, \tau_{i}\right)$, it will then follow that $Z\left(\sigma, \tau_{i+1}\right)$ is contained in $c\left[X\left(\sigma, \tau_{i+1}\right)\right]$, as required.

First we show that $F$ is $(k-2)$-dimensional. Denote the length $(k-1)$ element $\left[R\left(\tau_{i+1}\right)\right](\sigma)$ by $\sigma^{\prime}$. Note that $\sigma^{\prime} \leq R\left(\tau_{i+1}\right) \gamma$ so that $M\left(\sigma^{\prime}\right) \subset \mu\left(\tau_{i+1}\right)^{\perp}$. Apply the procedures of Sections 5 and 6 to $\sigma^{\prime}$ (i) to obtain a simple system $\left\{\delta_{1}^{\prime}, \delta_{2}^{\prime}, \ldots, \delta_{k-1}^{\prime}\right\}$ for the set $P_{\sigma^{\prime}}$ of positive roots in $M\left(\sigma^{\prime}\right)$ and (ii) to calculate the reordering $\left\{\theta_{1}^{\prime}, \theta_{2}^{\prime}, \ldots, \theta_{k-1}^{\prime}\right\}$ of the set $\left\{\epsilon_{1}^{\prime}, \epsilon_{2}^{\prime}, \ldots, \epsilon_{k-1}^{\prime}\right\}$ for which

$$
\sigma^{\prime}=R\left(\theta_{k-1}^{\prime}\right) \ldots R\left(\theta_{2}^{\prime}\right) R\left(\theta_{1}^{\prime}\right)
$$

where $\theta_{1}^{\prime} \prec \theta_{2}^{\prime} \prec \ldots \prec \theta_{k-1}^{\prime}$ and where $\epsilon_{j}^{\prime}$ is given by

$$
\epsilon_{j}^{\prime}=\left[R\left(\delta_{1}^{\prime}\right) R\left(\delta_{2}^{\prime}\right) \ldots R\left(\delta_{j-1}^{\prime}\right)\right]\left(\delta_{j}^{\prime}\right) \text { for } j=1, \ldots, k-1 .
$$

Since $\left\{\theta_{1}^{\prime}, \theta_{2}^{\prime}, \ldots, \theta_{k-1}^{\prime}\right\} \subset \mu\left(\tau_{i+1}\right)^{\perp}$, if we show that $\tau_{i+1}>\theta_{j}^{\prime}$ for $j=1, \ldots, k-1$, then it will follow that $\left\langle\theta_{1}^{\prime}, \theta_{2}^{\prime}, \ldots, \theta_{k-1}^{\prime}\right\rangle \in F$ and hence $F$ is $(k-2)$-dimensional.

Fix $j \in\{1, \ldots, k-1\}$. From $\epsilon_{j}^{\prime} \cdot \mu\left(\tau_{i+1}\right)=0$ we deduce that $\epsilon_{j}^{\prime} \neq \tau_{i+1}$ and that $R\left(\epsilon_{j}^{\prime}\right) \leq R\left(\tau_{i+1}\right) \gamma$. Therefore the length two element $\sigma^{\prime \prime}=R\left(\tau_{i+1}\right) R\left(\epsilon_{j}^{\prime}\right)$ precedes $\gamma$ and hence $\sigma$ by equation (3.4).

Assume now that $\epsilon_{j}^{\prime} \succ \tau_{i+1}$ and let $Q=P_{\sigma} \cap M\left(\sigma^{\prime \prime}\right)$. Then, as in the proof of Lemma 5.5, $\left\{\tau_{i+1}, \epsilon_{j}^{\prime}\right\}$ is the simple system for $Q$, with $\tau_{i+1}$ the first root and $\epsilon_{j}^{\prime}$ the last root. Thus $R\left(\tau_{i+1}\right) \epsilon_{j}^{\prime}$ is a positive root and $R\left(\tau_{i+1}\right) \epsilon_{j}^{\prime} \succ \tau_{i+1}$ (by Lemma 5.5).

Now consider $\left(\sigma^{\prime}\right)^{-1}\left(\epsilon_{j}^{\prime}\right)$, which is negative, by Proposition 7.1. However

$$
\left(\sigma^{\prime}\right)^{-1} \epsilon_{j}^{\prime}=\left(R\left(\tau_{i+1}\right) \sigma\right)^{-1} \epsilon_{j}^{\prime}=\sigma^{-1}\left(\left[R\left(\tau_{i+1}\right)\right]\left(\epsilon_{j}^{\prime}\right)\right) .
$$

Since $\left[R\left(\tau_{i+1}\right)\right]\left(\epsilon_{j}^{\prime}\right)$ is positive, Proposition 7.1 implies that $R\left(\tau_{i+1}\right) \epsilon_{j}^{\prime}=\epsilon_{a}$ for some $a$. Thus $\left[R\left(\tau_{i+1}\right)\right]\left(\epsilon_{j}^{\prime}\right)$ precedes $\tau_{i+1}$ (since each $\epsilon_{b}$ does), which contradicts the earlier conclusion that $\left[R\left(\tau_{i+1}\right)\right]\left(\epsilon_{j}^{\prime}\right) \succ \tau_{i+1}$. Thus the assumption that $\epsilon_{j}^{\prime} \succ \tau_{i+1}$ must have been false. 
Next we show that $|F|$ is spherically convex. Since $c\left[X\left(\sigma, \tau_{i}\right)\right]=Z\left(\sigma, \tau_{i}\right)$ by the inductive hypothesis, we obtain

$$
\begin{aligned}
|F| & =M\left(R\left(\tau_{i+1}\right) \sigma\right) \cap c\left[X\left(\sigma, \tau_{i}\right)\right] \cap S^{n-1} \\
& =M\left(R\left(\tau_{i+1}\right) \sigma\right) \cap Z\left(\sigma, \tau_{i}\right) \cap S^{n-1}
\end{aligned}
$$

which is convex.

Now $|V|$, being a cone with a convex $(k-2)$-dimensional base, must itself be convex and $(k-1)$-dimensional. The proof that $Z$ is contained in $c[V]$ involves a close examination of the facets of $c[V]$. First one argues that each facet of $c[V]$ is of the form $M(\sigma) \cap \mu\left(\tau_{j}\right)^{\perp}$ for some

$$
\tau_{j} \in\left\{\theta_{i_{1}}, \ldots, \theta_{i_{a}}\right\} \cup\left\{\tau_{i+1}, \tau_{j_{1}}, \ldots, \tau_{j_{b}}\right\},
$$

where $j_{l}>i+1$ for $l=1, \ldots, b$. (This step is similar to the corresponding argument for $c\left[V_{0}\right]$, with $\tau_{i+1}$ taking the place of $\theta_{k}$.) It then follows that

$$
\begin{aligned}
Z & =M(\sigma) \cap \mu\left(\theta_{1}\right)^{+} \cap \cdots \cap \mu\left(\theta_{k}\right)^{+} \cap \mu\left(\tau_{i+1}\right)^{+} \cap \mu\left(\tau_{i+2}\right)^{-} \cap \cdots \cap \mu\left(\tau_{t}\right)^{-} \\
& \subseteq M(\sigma) \cap \mu\left(\theta_{i_{1}}\right)^{+} \cap \cdots \cap \mu\left(\theta_{i_{a}}\right)^{+} \cap \mu\left(\tau_{i+1}\right)^{+} \cap \mu\left(\tau_{j_{i}}\right)^{-} \cap \cdots \cap \mu\left(\tau_{j_{b}}\right)^{-} \\
& =c[V] \quad \text { as required. } \square
\end{aligned}
$$

Corollary 7.7. For each $\sigma \leq \gamma$ the set $|X(\sigma)|$ is spherically convex. Furthermore, $|X(\sigma)|$ is the intersection with $S^{n-1}$ of the positive cone on the set $P_{\sigma}$ and we have

$$
|X(\sigma)|=S^{n-1} \cap M(\sigma) \cap \mu\left(\theta_{1}\right)^{+} \cap \cdots \cap \mu\left(\theta_{k}\right)^{+} .
$$

Proof. Apply Proposition 7.6 with $i=t$.

We are now in a position to prove that $[I, \gamma]$ is a lattice.

Theorem 7.8. If $W$ is a finite real reflection group equipped with the partial order $\leq$ defined by reflection length and $\gamma$ is a Coxeter element for $W$, then the interval $[I, \gamma]$ is a lattice.

Proof. Choose a simple system and Coxeter element $\gamma$ for $W$ as in Section 3. For $\sigma \leq \gamma$ and $\sigma \neq I$, construct the simplicial complex $X(\sigma)$ and define $X(I)$ to be the empty set $\emptyset$. We have seen that $X(\sigma)$ has dimension $l(\sigma)-1$ and its vertex set is

$$
P_{\sigma}=\left\{\rho_{i} \mid 1 \leq i \leq n h / 2 \text { and } R\left(\rho_{i}\right) \leq \sigma\right\} .
$$

Furthermore, $X(\sigma)$ is a subcomplex of $X(\gamma)$ and, by Corollary 7.7, $|X(\sigma)|$ is spherically convex.

Assume now that $\alpha$ and $\beta$ both precede $\gamma$. Then $X(\alpha) \cap X(\beta)$ is a sub-complex of $X(\gamma)$ and, since $|X(\alpha) \cap X(\beta)|=|X(\alpha)| \cap|X(\beta)|$, the set $|X(\alpha) \cap X(\beta)|$ is convex by Corollary 7.7. If $d$ denotes the dimension of $X(\alpha) \cap X(\beta)$, then $|X(\alpha) \cap X(\beta)|$ is a union of $d$-dimensional simplices and, for each $d$-simplex $\left\langle v_{0}, \ldots, v_{d}\right\rangle$ in $X(\alpha) \cap X(\beta)$, the linear subspaces $\operatorname{span}\left(\left\{v_{0}, \ldots, v_{d}\right\}\right)$ and $\operatorname{span}(X)$ coincide. We associate an element $\sigma \leq \gamma$ to $X(\alpha) \cap X(\beta)$ as follows. Choose a $d$-simplex $\left\langle v_{0}, \ldots, v_{d}\right\rangle \in X$ and assume (without loss of generality) that $v_{0} \prec v_{1} \prec \cdots \prec v_{d}$. Then

$$
R\left(v_{0}\right) R\left(v_{1}\right) \ldots R\left(v_{d}\right) \leq \gamma^{-1} \text { by definition of } X(\gamma),
$$

and we define $\sigma$ to be $R\left(v_{d}\right) \ldots R\left(v_{1}\right) R\left(v_{0}\right)$. Since $\operatorname{span}\left(v_{0}, \ldots, v_{d}\right)=M(\sigma)=$ $\operatorname{span}(X(\alpha) \cap X(\beta))$, it follows that $M(\sigma)$ contains and is spanned by the vertex set of $X(\alpha) \cap X(\beta)$. However this vertex set is $P_{\alpha} \cap P_{\beta}$, and the theorem now follows by our remarks in Section 2 . 


\section{Relationship With GENERALised ASSOCIAHEDRA}

In this section we embed the complex $X(\gamma)$ in a larger simplicial complex $E X(\gamma)$ whose vertex set consists of all positive roots and the negatives of the simple roots, and we show that the geometric realisation of $E X(\gamma)$ is a sphere. If $W$ is crystallographic, then we show that $E X(\gamma)$ coincides with the simplicial generalised associahedron for $W$ (defined in [13]).

Recall that $S_{1}=\left\{\alpha_{1}, \ldots, \alpha_{s}\right\}$ and $S_{2}=\left\{\alpha_{s+1}, \ldots, \alpha_{n}\right\}$ is a partition of the set of simple roots into two orthonormal sets. We will use the notation

$$
-S_{1}=\left\{-\alpha_{1}, \ldots,-\alpha_{s}\right\} \text { and }-S_{2}=\left\{-\alpha_{s+1}, \ldots,-\alpha_{n}\right\} .
$$

We note that the subscripting on the $\rho$ 's can be applied to negative indices with the convention that $\rho_{-k}=\rho_{n h-k}$.

Definition 8.1. We define a set, $E X=E X(\gamma)$, of simplices by declaring that

- the vertex set is the ordered set $\left\{\rho_{i} \mid-n+s+1 \leq i \leq n h / 2+s\right\}$,

- an edge joins $\rho_{i}$ to $\rho_{j}$ whenever $R\left(\rho_{i}\right) R\left(\rho_{j}\right) \leq \gamma^{-1}, i<j$ and $\rho_{i} \neq-\rho_{j}$, and

- $\left\langle\rho_{i_{1}}, \rho_{i_{2}}, \ldots, \rho_{i_{k}}\right\rangle$ forms a $(k-1)$-simplex if the vertices are distinct and pairwise joined by edges.

We note that it follows from the definitions that the simplices of $X(\gamma)$ are simplices of $E X(\gamma)$. The extra vertices are precisely the negatives of the simple roots $\left\{\alpha_{1}, \ldots, \alpha_{n}\right\}$. First, for $k=1, \ldots, n-s, \rho_{s+k}=-\gamma\left(\alpha_{s+k}\right)$ implies that $\rho_{-n+s+k}=-\alpha_{s+k}$. Secondly the set $\left\{\rho_{n h / 2+1}, \ldots, \rho_{n h / 2+s}\right\}$ is a permutation of $\left\{-\alpha_{1}, \ldots,-\alpha_{s}\right\}$.

Theorem 8.2. $E X(\gamma)$ is a simplicial complex and $|E X(\gamma)|$ is a sphere of dimension $n-1$.

Proof. Let $C$ be the spherical cross-polytope whose set of vertices is $\left\{ \pm \alpha_{1}, \ldots, \pm \alpha_{n}\right\}$. Then $C$ is a simplicial complex whose geometric realisation $|C|$ is the unit sphere in $\mathbf{R}^{n}$. We will show that $E X(\gamma)$ is a simplicial subdivision of $C$.

Consider the simplicial subdivision $C^{\prime}$ of $C$ which is defined as follows. Let $K$ be the sub-complex of $C$ which consists of the simplex $A=\left\langle\alpha_{1}, \ldots, \alpha_{n}\right\rangle$ and all its faces. We proved earlier that $|X(\gamma)|=|K|$. Thus $X(\gamma)$ is a simplicial subdivision of $K$.

Extend this subdivision to the rest of $C$ as follows. Any simplex of $C$ whose vertices are contained in $\left\{-\alpha_{1}, \ldots,-\alpha_{n}\right\}$ is not subdivided. If $B$ is a simplex of $C$ which contains both positive and negative roots, then we can write

$$
B=\left\langle\alpha_{i_{1}}, \ldots, \alpha_{i_{p}}\right\rangle *\left\langle-\alpha_{j_{1}}, \ldots,-\alpha_{j_{q}}\right\rangle
$$

where $i_{1}<\cdots<i_{p}, j_{1}<\cdots<j_{q}$, * denotes the spherical join, and $\left\{i_{1}, \ldots, i_{p}\right\}$ and $\left\{j_{1}, \ldots, j_{p}\right\}$ are disjoint. We extend the subdivision of the simplex $\left\langle\alpha_{i_{1}}, \ldots, \alpha_{i_{p}}\right\rangle$ (in $X(\gamma)$ ) by taking the join of this subdivision with the simplex $\left\langle-\alpha_{j_{1}}, \ldots,-\alpha_{j_{q}}\right\rangle$.

Suppose $J \subset\{1, \ldots, s\}, K \subset\{s+1, \ldots, n\}$ and that $\left\{\rho_{i_{1}}, \ldots, \rho_{i_{a}}\right\}$ is an ordered set of positive roots. Then $\left\{-\alpha_{j} \mid j \in J \cup K\right\} \cup\left\{\rho_{i_{1}}, \ldots, \rho_{i_{a}}\right\}$ is the vertex set for a simplex in $C^{\prime}$ or in $E X(\gamma)$ if and only if

$$
R\left(\rho_{i_{1}}\right) \ldots R\left(\rho_{i_{a}}\right) \leq\left(\prod_{l \in K} R\left(\rho_{l}\right)\right) \gamma^{-1}\left(\prod_{m \in J} R\left(\rho_{m}\right)\right) .
$$

It now follows that $C^{\prime}=E X(\gamma)$ as required. 
For convenience we recall some facts from [13] and express them in a manner consistent with our earlier notation. If $W$ is a crystallographic finite reflection group, then the simplicial generalised associahedron, $G A(W)$, for the simple system $S_{1} \cup S_{2}$ is a simplicial complex whose set of vertices, denoted $\Omega_{\geq-1}$, consists of all the positive roots and the negative simple roots. Two piecewise-linear involutions $\tau_{+}$and $\tau_{-}$are introduced in [13]. It can be shown that they are determined by

$$
\begin{gathered}
\tau_{+}(\beta)=\left\{\begin{array}{cc}
{\left[R_{1} R_{2} \ldots R_{s}\right](\beta)} & \text { if } \beta \notin-S_{2}, \\
\beta & \text { if } \beta \in-S_{2},
\end{array}\right. \\
\tau_{-}(\beta)=\left\{\begin{array}{cc}
{\left[R_{s+1} R_{s+2} \ldots R_{n}\right](\beta)} & \text { if } \beta \notin-S_{1}, \\
\beta & \text { if } \beta \in-S_{1} .
\end{array}\right.
\end{gathered}
$$

From this we deduce that the action of $\tau_{+} \tau_{-}$on $\Omega_{\geq-1}$ is given by

$$
\tau_{+} \tau_{-}(\beta)=\left\{\begin{array}{cc}
\gamma(\beta) & \text { if } \beta \notin\left(-S_{1}\right) \cup S_{2}, \\
-\beta & \text { if } \beta \in\left(-S_{1}\right) \cup S_{2},
\end{array}\right.
$$

and the action of the inverse $\tau_{-} \tau_{+}$is given by

$$
\tau_{-} \tau_{+}(\beta)=\left\{\begin{array}{cl}
\gamma^{-1}(\beta) & \text { if } \beta \notin\left(-S_{2}\right) \cup S_{1} \\
-\beta & \text { if } \beta \in\left(-S_{2}\right) \cup S_{1} .
\end{array}\right.
$$

The compatibility degree $(\alpha \| \beta)$ of any two elements $\alpha, \beta \in \Omega_{\geq-1}$ is characterised in [13] by the conditions

(i) $\left(-\alpha_{i} \| \alpha\right)=\max \left\{\left[\alpha: \alpha_{i}\right], 0\right\}$, where $\left[\alpha: \alpha_{i}\right]=\alpha \cdot \mu_{i}$ for $i=1, \ldots, n$, and

(ii) $(\alpha \| \beta)=\left(\tau_{ \pm} \alpha \| \tau_{ \pm} \beta\right)$ for all $\alpha$ and $\beta$.

We recall that two vertices $\alpha$ and $\beta$ in $G A(W)$ are connected by an edge if and only if $(\alpha \| \beta)=0$. Finally, we note that $G A(W)$ is completely determined by its one-skeleton.

Theorem 8.3. If $W$ is crystallographic, then $E X(\gamma)$ coincides with the simplicial generalised associahedron $G A(W)$.

Proof. We continue to use the earlier notation. First note that the vertex set $\Omega_{\geq-1}$ of the associahedron is the same as that of $E X(\gamma)$. Since each of $E X(\gamma)$ and $G A(W)$ is determined by its one-skeleton, it suffices to show that two vertices $\alpha$ and $\beta$ are joined by an edge in $E X(\gamma)$ if and only if they are joined by an edge in $G A(W)$. To simplify notation we will write $\rho_{i} \stackrel{G A}{\rightarrow} \rho_{j}$ or $\rho_{i} \stackrel{E X}{\rightarrow} \rho_{j}$ if $\rho_{i}$ and $\rho_{j}$ are connected by an edge in the simplicial complex $G A(W)$ or $E X(\gamma)$ respectively. Note that the ordering on the vertices of $E X(\gamma)$ is such that they are arranged into the following sets in the given order:

$$
-S_{2}, S_{1}, \gamma\left(-S_{2}\right), \gamma\left(S_{1}\right), \gamma^{2}\left(-S_{2}\right), \ldots, \gamma^{-1}\left(-S_{1}\right), S_{2},-S_{1}
$$

Suppose $\rho_{i} \prec \rho_{j}$. Then $\rho_{i} \stackrel{E X}{\rightarrow} \rho_{j}$ is equivalent to $R\left(\rho_{i}\right) R\left(\rho_{j}\right) \leq \gamma^{-1}$. First we can assume $\rho_{j} \neq-\rho_{i}$ since neither complex contains an edge from $\rho_{i}$ to $-\rho_{i}$.

Case 1. If $\rho_{i} \in-S_{2}$, then $\rho_{i}=-\alpha_{p}$ for some $p$ satisfying $s+1 \leq p \leq n$. Then $\rho_{i} \stackrel{G A}{\longrightarrow} \rho_{j}$ if and only if $\left(-\alpha_{p} \| \rho_{j}\right)=0$. However, by equation (3.4), this is equivalent to $R\left(\rho_{j}\right) \leq R\left(\alpha_{1}\right) \ldots R\left(\alpha_{p-1}\right) R\left(\alpha_{p+1}\right) \ldots R\left(\alpha_{n}\right)$. But, since $p>s$, we have $R\left(\alpha_{1}\right) \ldots R\left(\alpha_{p-1}\right) R\left(\alpha_{p+1}\right) \ldots R\left(\alpha_{n}\right)=\gamma R\left(\alpha_{p}\right)$. Thus $\rho_{i} \stackrel{G A}{\rightarrow} \rho_{j}$ is equivalent by equation (3.7) to $R\left(\rho_{j}\right) R\left(\rho_{i}\right) \leq \gamma$ which in turn is the criterion for $\rho_{i} \stackrel{E X}{\rightarrow} \rho_{j}$. 
Case 2. If $\rho_{i} \in S_{1}$, then $\rho_{i}=\alpha_{p}$, for some $p$ satisfying $1 \leq p \leq s$ and $\tau_{-} \tau_{+}\left(\rho_{i}\right)=-\rho_{i}=-\alpha_{p} \in-S_{1}$.

Case 2(a). If $\rho_{j}$ is also in $\in S_{1}$, then $\tau_{-} \tau_{+}\left(\rho_{j}\right)=-\rho_{j} \in-S_{1}$, and applying $\tau_{-} \tau_{+}$to both roots gives

$$
\left(\rho_{i} \| \rho_{j}\right)=\left(-\rho_{i} \|-\rho_{j}\right)=0
$$

since $\rho_{j}$ and $\rho_{i}$ are distinct simple roots. By definition of edges in the generalised associahedron we must have $\rho_{i} \stackrel{G A}{\rightarrow} \rho_{j}$. However, in this subcase, $\rho_{j}=\alpha_{q}$ with $1 \leq p<q \leq s$, so that

$$
R\left(\rho_{j}\right) R\left(\rho_{i}\right)=R\left(\rho_{i}\right) R\left(\rho_{j}\right) \leq \gamma
$$

which is again equivalent to the criterion for $\rho_{i} \stackrel{E X}{\rightarrow} \rho_{j}$.

Case 2(b). If $\rho_{j} \notin S_{1}$, then $j>s$, and we know that $\tau_{-} \tau_{+}\left(\rho_{j}\right)=\gamma^{-1}\left(\rho_{j}\right)$. Now $\rho_{i} \stackrel{G A}{\rightarrow} \rho_{j}$ if and only if $\left(-\alpha_{p} \| \gamma^{-1}\left(\rho_{j}\right)\right)=0$. However this is equivalent by equation (3.4) to

$$
R\left[\gamma^{-1}\left(\rho_{j}\right)\right] \leq R\left(\alpha_{1}\right) \ldots R\left(\alpha_{p-1}\right) R\left(\alpha_{p+1}\right) \ldots R\left(\alpha_{n}\right) .
$$

Since $1 \leq p \leq s$, we have $R\left(\alpha_{1}\right) \ldots R\left(\alpha_{p-1}\right) R\left(\alpha_{p+1}\right) \ldots R\left(\alpha_{n}\right)=R\left(\alpha_{p}\right) \gamma$. Thus $\rho_{i} \stackrel{G A}{\longrightarrow} \rho_{j}$ is equivalent to $R\left(\rho_{i}\right) R\left[\gamma^{-1}\left(\rho_{j}\right)\right] \leq \gamma$ by equation (3.7). But

$$
R\left(\rho_{i}\right) R\left[\gamma^{-1}\left(\rho_{j}\right)\right] \leq \gamma \Leftrightarrow R\left(\rho_{i}\right) \leq \gamma R\left[\gamma^{-1}\left(\rho_{j}\right)\right]=R\left(\rho_{j}\right) \gamma,
$$

using equations (3.7) and (3.2). The last condition in turn is the criterion for $\rho_{i} \stackrel{E X}{\longrightarrow} \rho_{j}$

Case 3. If $i>s$ let $m$ be the smallest positive integer with the property that

$$
\left(\tau_{-} \tau_{+}\right)^{m}\left(\rho_{i}\right) \in\left(-S_{2}\right) \cup S_{1} .
$$

Now $\rho_{i} \stackrel{G A}{\rightarrow} \rho_{j}$ if and only if $\left(\tau_{-} \tau_{+}\right)^{m}\left(\rho_{i}\right) \stackrel{G A}{\longrightarrow}\left(\tau_{-} \tau_{+}\right)^{m}\left(\rho_{j}\right)$. However, Case 1 or Case 2 now applies to this new pair of roots and $\left(\tau_{-} \tau_{+}\right)^{m}=\gamma^{-m}$ when applied to $\rho_{i}$ and $\rho_{j}$. Thus $\rho_{i} \stackrel{G A}{\longrightarrow} \rho_{j}$ if and only if

$$
R\left(\gamma^{-m}\left(\rho_{j}\right)\right) R\left(\gamma^{-m}\left(\rho_{i}\right)\right) \leq \gamma
$$

by the proof in cases 1 and 2. But this is equivalent to $R\left(\gamma^{-m}\left(\rho_{i}\right)\right) \leq R\left(\gamma^{-m}\left(\rho_{j}\right)\right) \gamma$ or $\rho_{i-m n} \cdot \mu_{j-m n}=0$. Since $\gamma$ is an isometry this is equivalent to $\rho_{i} \cdot \mu_{j}=0$ and hence to $\rho_{i} \stackrel{E X}{\rightarrow} \rho_{j}$.

Note 8.4. We observe that Theorem 8.3 provides a new proof that the simplicial generalised associahedron is a simplicial complex whose geometric realisation is a sphere. This proof is independent of the classification of finite real reflection groups and extends the work of Fomin and Zelevinsky [13] to include the non-crystallographic finite reflection groups. Furthermore Definition 8.1 and Theorem 8.3 characterise the notion of compatibility (in the sense of Section 3.2 of [13]) in terms of non-crossing partitions.

\section{ACKNOWLEDGEMENTS}

We thank the referee for many helpful suggestions and comments. 


\section{REFERENCES}

[1] C.A. Athanasiadis and V. Reiner, Noncrossing partitions for the group $D_{n}$, SIAM J. Discrete Math. 18 (2004), 397-417. MR2112514 (2006b:06004)

[2] D. Bessis, F. Digne, J. Michel, Springer theory in braid groups and the Birman-Ko-Lee monoid, Pacific J. Math. 205 (2002), no. 2, 287-309. MR1922736 (2003f:20054)

[3] D. Bessis, The dual braid monoid, Ann. Sci. École Norm. Sup.(4), (2003), no. 36(5), 647-683. MR2032983 (2004m:20071)

[4] M. Bestvina, Non-positively curved aspects of Artin groups of finite type. Geom. Topol. 3 (1999), 269-302. MR1714913 (2000h:20079)

[5] J. Birman, K. Y. Ko, S. J. Lee, A new approach to the word and conjugacy problems in the braid groups. Adv. Math. 139 (1998), no. 2, 322-353. MR1654165 (99m:20082)

[6] N. Bourbaki, Lie groups and Lie algebras. Chapters 4-6. Elements of Mathematics (Berlin). Springer-Verlag, Berlin, 2002. MR1890629 (2003a:17001)

[7] T. Brady, Artin groups of finite type with three generators, Michigan Math. J. 47 (2000), no. 2, 313-324. MR1793627 (2001j:20056)

[8] T. Brady, A partial order on the symmetric group and new $K(\pi, 1)$ 's for the braid groups, Adv. Math. 161 (2001), no. 1, 20-40. MR1857934 (2002k:20066)

[9] T. Brady, C. Watt, A partial order on the orthogonal group, Comm. Algebra 30 (2002), no. 8, 3749-3754. MR1922309 (2003h:20083)

[10] T. Brady, C. Watt, $K(\pi, 1)$ 's for Artin groups of finite type, Proceedings of the Conference on Geometric and Combinatorial Group Theory, Part I (Haifa, 2000). Geom. Dedicata 94 (2002), 225-250. MR1950880 (2004i:20066)

[11] R. Charney, J. Meier, K. Whittlesey, Bestvina's normal form complex and the homology of Garside groups. Geometriae Dedicata,(2004) no. 105, 171-188. MR2057250 (2005e:20057)

[12] M.J. Dyer, Hecke algebras and shellings of Bruhat intervals. Compositio Math. 89 (1993), no. 1, 91-115. MR1248893 (95c:20053)

[13] S. Fomin, A. Zelevinsky, Y-systems and generalised associahedra, Ann. Math. (2003), no. 158, 977-1018. MR2031858 (2004m:17010)

[14] F. A. Garside, The braid group and other groups, Quart. J. Math. Oxford (2), 20 (1969), 235-254. MR0248801 (40:2051)

[15] R. Kane, Reflection Groups and Invariant Theory. Springer SMC Series, Springer-Verlag, New York, 2001. MR1838580 (2002c:20061)

[16] G. Kreweras, Sur les partitions non croisees d'un cycle, Disc. Math. 1 333-350 (1972). MR0309747 (46:8852)

[17] V. Reiner, Non-crossing partitions for classical reflection groups, Discrete Math. 177 (1997), 195-222. MR1483446 (99f:06005)

[18] R. Steinberg, Finite reflection groups. Trans. Amer. Math. Soc. 91 (1959) 493-504. MR0106428 (21:5160)

School of Mathematical Sciences, Dublin City University, Glasnevin, Dublin 9, IreLAND

E-mail address: tom.brady@dcu.ie

School of Mathematical Sciences, Dublin Institute of Technology, Kevin St., Dublin

8, IRELAND

E-mail address: colum.watt@dit.ie 